\title{
One-Pot Seed-Mediated Growth of Co Nanoparticles by the Polyol Process: Unraveling the Heterogeneous Nucleation
}

Raj Kumar Ramamoorthy, ${ }^{1}$ Arnaud Viola, ${ }^{2}$ Bilel Grindi, ${ }^{1}$ Jennifer Peron, ${ }^{2}$ Christophe Gatel, ${ }^{3}$ Martin Hytch, ${ }^{3}$ Raul Arenal, ${ }^{4,5}$ Lorette Sicard, ${ }^{2}$ Marion Giraud, ${ }^{2}$ Jean-Yves Piquemal, ${ }^{2,}$, Guillaume Viau $^{1, *}$

${ }^{1}$ Université de Toulouse, UMR 5215 INSA, CNRS, UPS, Laboratoire de Physique et Chimie des NanoObjets, 135 avenue de Rangueil F-31077 Toulouse cedex 4, France

${ }^{2}$ ITODYS, Sorbonne Paris Cité, Université Paris Diderot, 15 rue Jean-Antoine de Baif, 75013 Paris, France

${ }^{3}$ Centre d'Elaboration de Matériaux et d'Etudes Structurales, CEMES-CNRS, 29 rue Jeanne Marvig, B.P. 94347, 31055 Toulouse, France

${ }^{4}$ Laboratorio de microscopias avanzadas (LMA), Instituto de Nanociencia de Aragon (INA), U. Zaragoza, C/ Mariano Esquillor s/n, 50018 Zaragoza, Spain

${ }^{5}$ ARAID Foundation, 50018 Zaragoza, Spain

Dedicated to Professor Fernand Fiévet on the occasion of his 75th birthday

\begin{abstract}
The one-step seed-mediated synthesis is widely used for the preparation of ferromagnetic metal nanoparticles (NPs) since it offers a good control of particle morphology. Nevertheless, this approach suffers from a lack of mechanistic studies because of the difficulties to follow in real time the heterogeneous nucleation and to predict structure effects with seeds that are generated in situ. Here, we propose a complete scheme of the hetero-nucleation process involved in onepot seed-mediated syntheses of cobalt nanoparticles in liquid polyols, relying on geometrical phase analysis (GPA) of high-resolution HAADF-STEM images and in situ measurements of the molecular hydrogen evolution. Cobalt particles of different shapes (rods, platelets or hourglass like particles) were grown by reducing cobalt carboxylate in liquid polyols in presence of iridium or ruthenium chloride as nucleating agent. A reaction scheme was established by monitoring the $\mathrm{H}_{2}$ evolution resulting from the decomposition of metal hydrides, formed in situ by $\beta$-elimination of metal alkoxides, and from the polyol dehydrogenation, catalytically activated by the metal particles. This is a very good probe for both the noble metal nucleation and the heterogeneous
\end{abstract}


nucleation of cobalt, showing a good separation of these two steps. Ir and Ru seeds with a size in the range 1-2 nm were found exactly in the center of the cobalt particles, whatever the cobalt particle shape, and high-resolution images revealed an epitaxial growth of the hcp Co on fcc Ir or hcp Ru seeds. The microstructure analysis around the seeds evidenced two different ways of relaxing the lattice mismatch between the seeds and the cobalt, with the presence of dislocations around the Ir seeds and compression zones of the cobalt lattice near the Ru seeds. The relationship between the nature of the nucleating agent, the reaction steps and the microstructure is discussed.

KEYWORDS: Cobalt nanoparticles, Polyol synthesis, nucleation, HAADF-STEM, geometric phase analysis.

\section{INTRODUCTION}

Immense progresses have been done over the past 20 years to synthesize metal nanoparticles (NPs) by liquid phase process with a precise control of the size and shapes opening wide fields of applications in magnetism, ${ }^{1}$ optics, ${ }^{2}$ electronics ${ }^{3}$ and selective catalysis. ${ }^{4}$ The various synthetic strategies include the use of capping agents, chemical etching, templating with porous materials, etc. ${ }^{5}$ To circumvent the difficulty associated with the separation of nucleation and grow steps and obtaining well controlled nanoparticles, an elegant strategy consists in using seeds, added in the reaction mixture or generated in situ, that act as the nucleating agent for the second metal. ${ }^{6}$ This so-called seed-mediated growth (SMG) approach has been widely used to prepare core-shell materials, ${ }^{7}$ to increase ${ }^{8}$ or to decrease $e^{9,10,11,12}$ in a controlled manner the particle mean size, to grow anisotropic particles, ${ }^{13,14}$ and to synthesize metal particles with unusual structure and shape thanks to an epitaxial growth on facetted seeds. ${ }^{15}$ The general principle of the SMG method is that the particles grow at concentration below supersaturation since the energy barrier related to heterogeneous nucleation is lower than that of homogeneous nucleation. ${ }^{6,16}$ Therefore, the particle size depends on the seeds number injected in the medium while the particle shape and structure can be finely tuned playing upon the seed structure.

Experimentally, the precise knowledge of the heterogeneous nucleation mechanism associated to a fine control of the seed-mediated growth methods requires to tackle three issues: (i) the localization of the seed inside the final particles, (ii) the structural characterization of the interface between the seed and the second metal, and finally (iii) the in situ monitoring of the heterogeneous nucleation to determine the kinetic of nucleation and growth. Scanning 
transmission electron microscopy coupled with high angle annular dark field imaging (HAADFSTEM) is extensively used when the seed and the particle have a large Z-contrast. ${ }^{17}$ Seeds located exactly in the middle of the final particles resulting from a symmetrical growth were observed in several cases, including cubic ${ }^{17}$ or cuboid particles, ${ }^{18}$ bipyramids ${ }^{19}$ or tetrapods. ${ }^{20}$ In some cases, a symmetry breaking was observed, as in $\mathrm{Ag}$ or $\mathrm{Cu}$ nanorods that grow only on one side of $\mathrm{Pd}$ decahedral seeds through a mechanism favored by a slow reduction rate and/or a large lattice mismatch. ${ }^{21,22}$ In some seed-mediated syntheses, no foreign seeds were found inside the final particles showing, in these cases, that the seeds act as catalysts for the homogenous nucleation rather than as template for the heterogenous nucleation. ${ }^{23}$ The structural characterizations by high-resolution transmission electron microscopy help to understand the heterogeneous nucleation. In case of binary Pt@Pd and Pt@Au nanocrystals prepared by epitaxial growth from a well-crystallized Pt seed, the lattice mismatch between the seed and the second metal is of paramount importance for the control of the final shape. ${ }^{17}$ Structural studies revealed also the role of twin faults in the growth mechanism of multiple twinned particles with different shapes. ${ }^{24,19}$ The in situ monitoring of the heterogeneous nucleation and the growth steps is probably the more challenging. When the chemical reaction allows, small angle x-ray scattering (SAXS) is a very useful in situ probe to follow the nucleation and growth steps. ${ }^{25,26}$ UV-visible-NIR absorption and surface-enhanced Raman scattering (SERS) were used, respectively, to study the kinetic of $\mathrm{Ag}$ growth on Au rods, ${ }^{18}$ and to monitor the overgrowth of a second metal on Ag seeds thanks to the addition of a molecule probe in the medium. ${ }^{27}$ Other indirect methods, such as the potentiometric titration of co-anions in turbid solutions ${ }^{28}$ or the catalytic activity toward olefin hydrogenation, ${ }^{29}$ were also used as in situ probes of nucleation and growth of metal nanoparticles.

Most of the mechanism studies on SMG syntheses described in the literature are related to noble metal particles using to two-steps protocol, i.e. synthesis of well-defined seeds and injection in the reacting medium. The two-steps SMG methods allow for an optimal control of the seeds size and shape and allow for homo- or hetero-epitaxy of the second metal onto the seeds. They are particularly well suited for growth mechanism studies and for an identification of the experimental parameters favoring either thermodynamic or kinetic control of the particle growth. ${ }^{30}$ To our knowledge, no mechanistic study was done on ferromagnetic metals, despite the fact that such methods were developed by several groups in the last decade. Among the different chemical solution routes for metal ferromagnetic nanoparticles synthesis, the polyol process is one of the favorite since it is cost-effective and easy to implement, the polyol acting both as the solvent and the reducing agent. ${ }^{31,32}$ One-pot in situ SMG, i.e. following a protocol where noble metal seeds are generated in situ from a noble metal precursor added concomitantly with the Co precursor at the beginning of the reaction, was developed by several groups to synthesize cobalt $33,34,35$ and cobalt-nickel ${ }^{36,37,38,39,40}$ nanorods or nanowires, as building blocks for 
permanent magnet materials $\mathrm{s}^{41,42}$ or as catalysts. ${ }^{33,43,44,45,46}$ The one-pot in situ SMG methods present the advantage to be scalable to several grams per batch ${ }^{42,47}$ but the drawback compared to the ex situ methods is the difficulty to study properly the nucleation steps as far as the seeds are not isolated. Several studies reported that the addition of a small amount of nucleating agent was mandatory to induce cobalt anisotropic growth and formation of nanowires, ${ }^{38,43,48,49}$ but despite this empirical support on the role of the noble metal, no clear mechanism of the seeding process was described.

In this paper, we present a complete description including the structural and chemical aspects of the heterogeneous nucleation process involved in the one-pot SMG polyol synthesis of cobalt nanoparticles with different shapes, relying both on high-resolution HAADF-STEM images and on an original method to follow in situ the nucleation and growth steps. Two nucleating agents were used, iridium or ruthenium chloride. Taking advantage of the high difference in atomic numbers between $\mathrm{Co}$ and the two noble metals, we were able to image for the first time Ru or Ir tiny seeds (mean size $c a$. 1-2 $\mathrm{nm}$ ) inside the cobalt nanoparticles. High-resolution HAADF-STEM images showed an epitaxial growth of hcp Co on both kind of seeds. Two different ways to relax the lattice mismatch between the seeds and the cobalt, with either the presence of dislocations or compressed zones near the interface between the noble metal and the cobalt, were evidenced by geometric phase analysis. The monitoring of the nucleation and growth steps by classical SAXS or UV-visible absorption studies being difficult because of the turbidity of the reaction mixtures, we have developed a methodology to follow in situ the nucleation steps by measuring the evolution of molecular hydrogen during the reaction, which allows us to distinguish the noble metal nucleation from the heterogeneous nucleation and growth of cobalt.

\section{RESULTS AND DISCUSSION}

Nanometer-sized cobalt particles were synthesized by the polyol process, using cobalt long-chain carboxylates, cobalt dodecanoate or cobalt palmitate, as precursors, and iridium or ruthenium chloride with different hydration states, as nucleating agents. Previous studies showed that cobalt particles with well defined shapes could be prepared by SMG in liquid polyols by adjusting the basicity of the medium ${ }^{50}$ and the concentration of long chain carboxylate ions, ${ }^{51}$ the shape homogeneity requiring also a good control of the heating ${ }^{50}$ and stirring rates. ${ }^{47}$ The growth of cobalt nanorods was ascribed to the preferential adsorption of the long-chain carboxylate ions on the $\{11 \overline{2} 0\}$ facets of the hcp cobalt nanocrystals. Platelets were prepared by increasing the long chain carboxylate ions concentration. Their formation is in agreement with the theoretical calculations that predicts the greater stabilization of the (0001) planes in the presence of a large excess of long chain carboxylate as capping agent. ${ }^{51}$ Cobalt particles with different shapes were synthesized following this strategy. The cobalt precursor was initially mixed with iridium or 
ruthenium chloride in basic solution of polyol (1,2 butanediol or 1,2 propanediol) and the suspension was heated to $175^{\circ} \mathrm{C}$ or $215^{\circ} \mathrm{C}$. The seeded cobalt particles were obtained after only few ten minutes at this temperature (see details in the experimental section). Transmission electron microscopy images of eight representative samples are presented in Figures S1-3 of supplementary information.

Cobalt nanorods noted $\mathrm{CO}_{\mathrm{Ir}}$ and $\mathrm{CO}_{\mathrm{Ru}}$ were obtained by reduction of cobalt dodecanoate in basic solution of 1,2-butanediol with respectively, $\mathrm{IrCl}_{3} \cdot \mathrm{xH}_{2} \mathrm{O}$, hydrated or anhydrous $\mathrm{RuCl}_{3}$, as seeding agent, following a procedure already described. ${ }^{35,50}$ The mean diameter of the CoIr rods was found equal to $15 \mathrm{~nm}$ and the mean aspect ratio to 7 (Figure S1a). The $\mathrm{CO}_{\mathrm{Ru}}$ nanorods prepared with hydrated ruthenium chloride exhibit almost the same mean diameter $\left(d_{m}=16 \mathrm{~nm}\right)$ and aspect ratio $\left(A R_{m}=8\right)$ (Figure $\left.S 1 b\right)$. Shorter $\mathrm{Co}_{\mathrm{Ru}}$ rods were obtained when anhydrous ruthenium chloride was used as nucleating agent, as previously reported. ${ }^{35}$ The mean length of these $\mathrm{Co}_{\mathrm{Ru}}$ nanorods was comprised between 45 and $50 \mathrm{~nm}$ and the mean aspect ratio between 5 and 3 depending on the heating conditions (Figure S1c-d). The thinner rods were obtained with the highest heating rate and the highest reaction temperature. Indeed, the rods prepared at $175^{\circ} \mathrm{C}$ exhibited a mean diameter of $15 \mathrm{~nm}$ (Figure S1b,d). When the reaction temperature was increased to $215^{\circ} \mathrm{C}$ thanks to a high-pressure reactor affording solvothermal conditions, the mean diameter was decreased to $10 \mathrm{~nm}$ (Figure S1c). X-ray diffraction (XRD) patterns showed that the Colr and $\mathrm{CO}_{\mathrm{Ru}}$ rods crystallize with the hcp structure with the crystallographic $c$ axis parallel to the long axis of the rods (Figure S4, supplementary information). This crystallographic orientation was confirmed by the high-resolution TEM images (Figure S5, supplementary information). Co Ru nano-hourglasses (Figure S2a) and $\mathrm{CO}_{\mathrm{Ru}}$ nanoplatelets (Figure S3a) were synthesized by reduction of cobalt dodecanoate in solutions of sodium dodecanoate in 1,2-butanediol and 1,2-propanediol, respectively, using in both cases anhydrous $\mathrm{RuCl}_{3}$ as the seeding agent. The same shapes, with a better homogeneity in terms of sizes and shapes, were obtained by reduction of cobalt palmitate in sodium palmitate solutions both diols (Figures S2b andS3b). The $\mathrm{CO}_{\mathrm{Ru}}$ nano-hourglasses crystallize with the hcp structure (Figure $\mathrm{S} 4$, supplementary information). The hcp $c$ axis is parallel to the 6-fold rotation axis of the hourglass, the basal planes are the (0001) planes and the diagonal faces of the crystals are the $\{11 \overline{2} l\}$ planes (Figure S6, supplementary information). A similar shape was already observed with hcp ruthenium particles synthesized using oleylamine as surfactant. ${ }^{52}$ This unusual morphology can be explained by a growth that begins along the c-axis and rapidly competes with growth perpendicular to it. The $\mathrm{CO}_{\mathrm{Ru}}$ nanoplatelets synthesized in a sodium palmitate solution in 1,2-propanediol crystallized mainly with the hcp structure, the $c$ axis being perpendicular to the platelets (Figure S2, supplementary information). This crystallographic orientation was confirmed by HRTEM (Figure S7, supplementary information). The large excess of long-chain carboxylate ions in 1,2-butanediol causes a significant increase of the surface area of the (0001) faces at the tips and decreases the particle aspect ratio. This effect is enhanced in 1,2-propanediol with the formation of platelets exhibiting mainly (0001) faces. 
Several remarks can be made about the influence of the nucleating agent, which had not been discussed so far. First, in the Co NR growth conditions no strong difference was observed between $\mathrm{IrCl}_{3} \cdot \mathrm{xH}_{2} \mathrm{O}$ and $\mathrm{RuCl}_{3} \cdot \mathrm{xH}_{2} \mathrm{O}$, despite the fact that $\mathrm{Ir}$ and $\mathrm{Ru}$ crystallize with a different structure, respectively hcp and fcc, with furthermore different lattice mismatch with hcp cobalt. Second, subtle but significant differences were observed between $\mathrm{RuCl}_{3} \cdot \mathrm{xH}_{2} \mathrm{O}$ and anhydrous $\mathrm{RuCl}_{3}$, the latter nucleating agent giving rods with smaller mean diameter and smaller aspect ratio. From these observations, the questions of (i) the role of the seed, of its size, shape and structure on the nucleation and subsequent growth of the cobalt anisotropic nanostructures and (ii) of the chemical reaction involved in the seed formation arise.

HAADF-STEM images of Colr rods are shown in Figure 1. The presence of a brighter zone located at the right center of the nanorods can be clearly observed (Figure 1a-c). Energy dispersive X-ray spectroscopy (EDS) of this precise area of the Colr rods confirmed the presence of Ir along with cobalt (Figure 1c), while no iridium could be detected in any other part of the rods (the brighter areas at the tips are due to a larger thickness). The observation of the hetero-nuclei is permitted by the high contrast between $C_{0}$ and $I r$ due to the large difference in their atomic number $\left(Z_{C_{0}}=\right.$ 27 and $\left.Z_{1 r}=77\right)$. In the approximation based on Rutherford scattering, HAADF-STEM image contrast of thin samples is proportional to the thickness and to $Z^{2}$, the power law observed experimentally is generally $Z^{\alpha}$ with $\alpha \sim 1.7 .{ }^{53}$ Thus, the intensity scattered by a Co atom is about $15 \%$ of the intensity scattered by an Ir atom. Thanks to this high contrast, it was possible to obtain for the first time the unambiguous proof of the existence and the location of the noble metal seed in anisotropic cobalt particles prepared by the polyol process. The comparison with bright field images on which the seeds can hardly be detected (Figure S8, supplementary information) emphasizes the interest of the dark field technique. HAADF-STEM images and EDS analysis showed that each particle contains a single Ir nucleus and it is noteworthy that the Ir seeds are located exactly in the middle of the rods. On Figure 1c, the rod length is of $176 \mathrm{~nm}$ and the distances between the seed and the two tips are of 87 and $89 \mathrm{~nm}$, while the rod diameter is of $15.2 \mathrm{~nm}$ and the center of the observed seed is located at $7.6 \pm 0.2 \mathrm{~nm}$ from the edges of the rod. The location of the seed exactly in the middle of the rods was confirmed on several examples. The size of the Ir seeds measured on several rods was found equal to $1.6 \pm 0.3 \mathrm{~nm}$. The technical performance must be emphasized here since in most of the previous studies the seeds observed inside metal particles by HAADF-STEM exhibited a size higher than $5 \mathrm{~nm}$. Few cobalt tripods were observed besides the rods. In these particles, the Ir seed is also found in the center of the particles, i.e. at the junction of the three rods (Figure S9, supplementary information). 

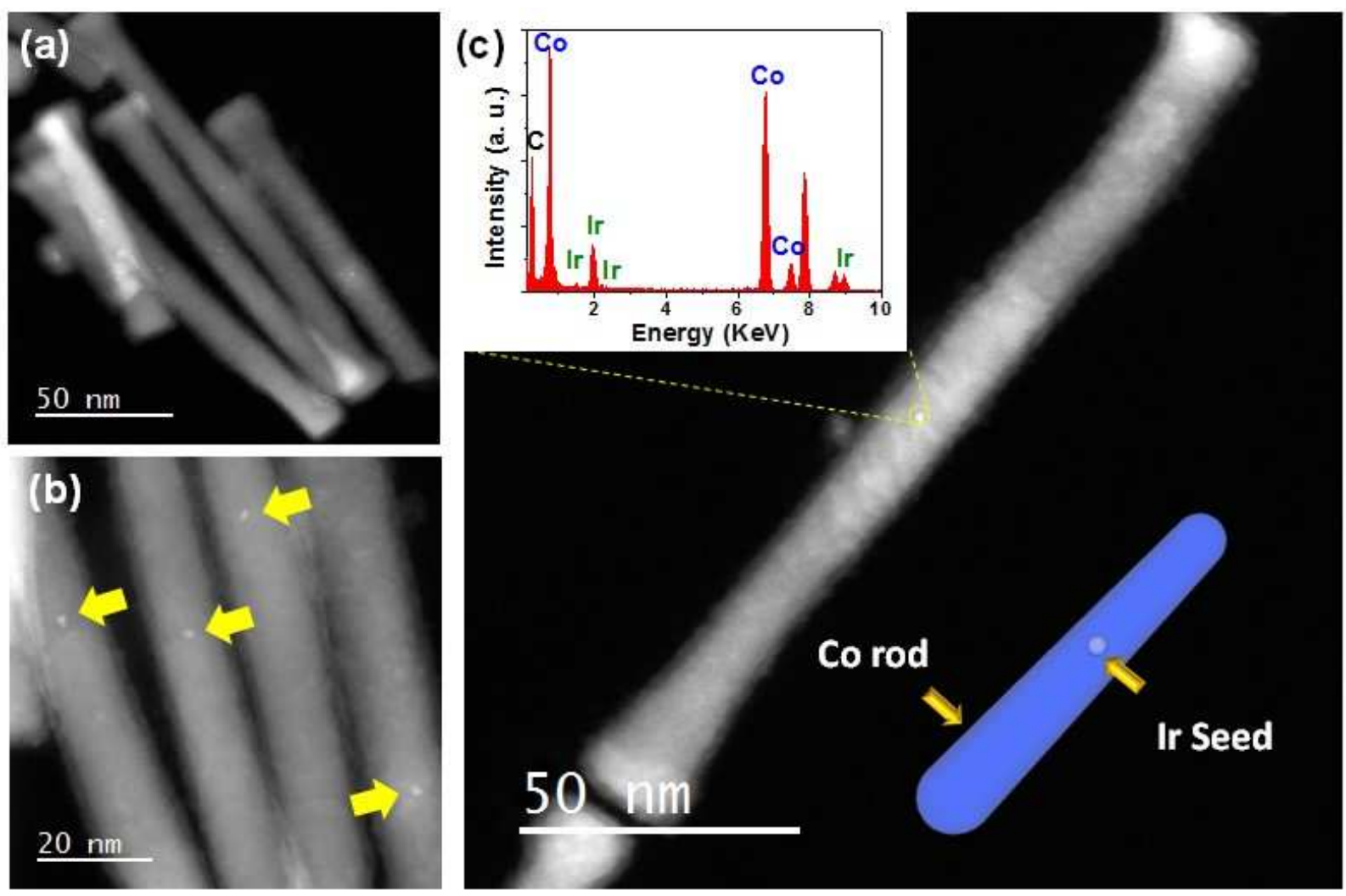

Figure 1. (a) HAADF-STEM image of cobalt nanorods prepared by the polyol process with $\mathrm{IrCl}_{3}$ as nucleating agent; (b) detail of the HAADF-STEM image, the yellow arrows are guide for the eyes showing the presence of a bright zone inside each rod; (c) EDS analysis of the bright zone at the center of a $\mathrm{CO}_{\text {Ir }}$ nanorod showing the presence of iridium (surface area analyzed $=1 \mathrm{~nm}^{2}$ ) and schematics illustrating the presence of a seed located in the center of each rod.

HAADF-STEM images of $\mathrm{CO}_{\mathrm{Ru}}$ nanoparticles with different shapes are shown in Figure 2. A bright zone was observed in the center of the platelets (Figure 2a) and rods (Figure 2b). EDS analysis evidenced the presence of Ru in these bright zones and its absence in other areas (Figure $2 \mathrm{c}$ and Figure S10, supplementary information). Besides the bright spots corresponding to Ru NPS located in the center, several bright zones also appear on HAADF-STEM images of the CORu platelets. This additional contrast is due to small islands of cobalt oxide, $\mathrm{CoO}$, evidenced by high resolution TEM that have grown epitaxially on the Co (0001) planes of the platelets during the particles washing and drying in air (Figure S7b, supplementary information). EDS analyses were very useful to clearly distinguish the Ru seeds from the $\mathrm{CoO}$ islands (Figures S11, supplementary information). The ratio $[\mathrm{Z}(\mathrm{Co}) / \mathrm{Z}(\mathrm{Ru})]^{1.7}$ is equal to $43 \%$, thus the $\mathrm{Z}$ contrast between $\mathrm{Co}$ and $\mathrm{Ru}$ is not very high and can be of same order of magnitude than the HAADF contrast of 1.5-2 nm thick $\mathrm{CoO}$ additional layers on each side of the Co platelets. In the case of the $\mathrm{CO}_{\mathrm{Ru}}$ nano-hourglass particles, it was very difficult to identify the tiny Ru seeds on the HAADF-STEM images because of the very bright signal scattered by the thick tips. Again, local energy dispersive spectroscopy at 
$\mathrm{nm}$ scale was very useful to locate the Ru seed. EDS profile determined th the Co $\mathrm{K}$ and Ru L edges along a central line allowed to evidence the Ru seed in the center of the particles (Figures $2 \mathrm{~d}$-e).

The Ru seeds in the nanorods do not exhibit a faceted shape. In Figure $2 b$, the Ru seeds have a size of $c a .1 .3 \times 1.8 \mathrm{~nm}$ and $2.2 \times 3.2 \mathrm{~nm}$. In other examples, the seeds are smaller (diameter between 1 and $1.5 \mathrm{~nm}$ ) and exhibit a rounded shape (Figure S12, supplementary information). The average size and standard deviation measured on several rods were found equal to $1.7 \pm 0.5$ $\mathrm{nm}$. The mean size of the Ru seeds inside the Co platelets was approximately $1.8 \mathrm{~nm}$ with a broad size distribution and the seed shape varied a lot from one platelet to another. Ultrasmall particles with rounded shape and a diameter between 1 and $1.5 \mathrm{~nm}$ but also crescent-shaped and starshaped particles that can extend over $3 \mathrm{~nm}$ were found (Figure S13, supplementary information).

High-resolution (HR) HAADF-STEM was performed to establish the relationship between the structure of the Ir and Ru seeds and that of the Co particles. HR HAADF-STEM images of Colr nanorods are presented in Figure 3. No discontinuity of the growth planes when they cross the seed can be noticed. As already mentioned above, the growth axis of the rods is the $c$ axis. Outside the Ir seed, the interplanar spacing is $2.02 \AA$ in agreement with the (0002) planes of bulk hcp Co. ${ }^{54}$ On top of the seed, the interplanar spacing is $2.16 \AA$ (Figure $3 \mathrm{~b}$ ) which is an average between the (0002) distance of hcp Co and the (111) distance of bulk fcc Ir, $2.217 \AA .{ }^{55}$ This average value results from the overlap of the Co and Ir lattice fringes. On both HR images of Figure 3, the emergence of several edge dislocations can be seen, appearing even more clearly on the Fourier filtered images (Figure 3d). These dislocations are located very close to the seeds, some at a distance lower than $1 \mathrm{~nm}$ (Figure 3a,c-d). In the supplementary information are reported other examples of HR HAADF-STEM images of CoIr nanorods: the crystallographic orientation is always the same as that of figure 3 but the structural defects around the Ir seeds are observed over the whole diameter of the rods (see Figure S14, supplementary information). 

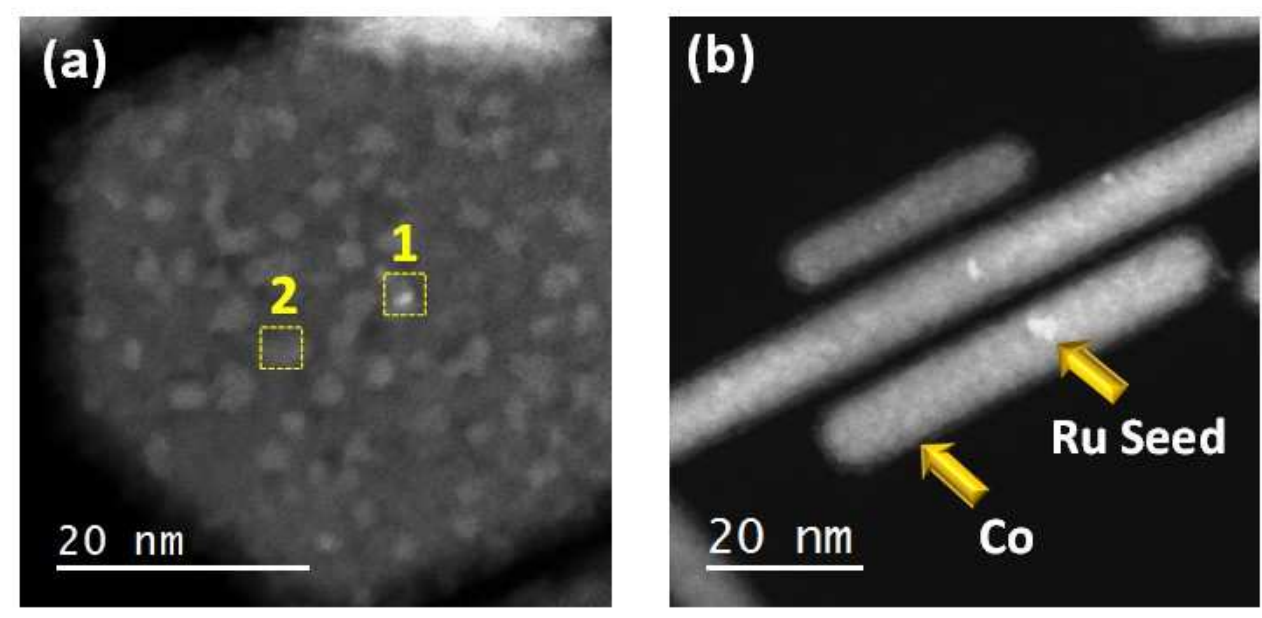

(c)
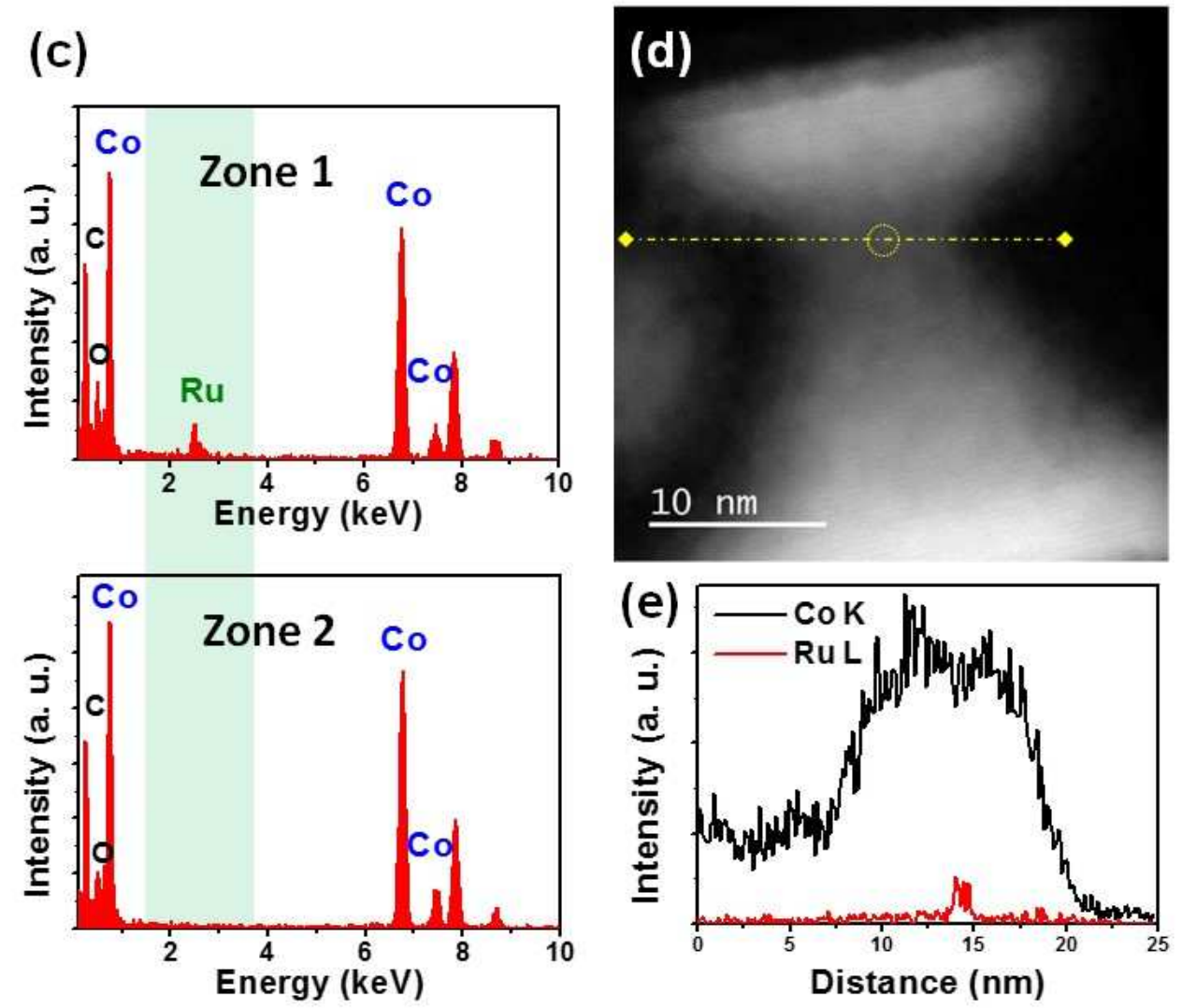

Figure 2. (a) HAADF-STEM image of a cobalt platelet prepared by the polyol process with $\mathrm{RuCl}_{3}$ as nucleating agent; (b) HAADF-STEM image of cobalt nanorods prepared by the polyol process with $\mathrm{RuCl}_{3}$ as nucleating agent; (c) EDS analysis of the bright zone at the center of the CoRu platelet (zone 1) and aside (zone 2) (surface area analyzed $=1 \mathrm{~nm}^{2}$ ); (d) HAADF-STEM image of a cobalt nano-hourglass particle prepared by the polyol process with $\mathrm{RuCl}_{3}$ as nucleating agent and (e) EDS profile along the yellow line determined at the Co K and Ru L edges. 

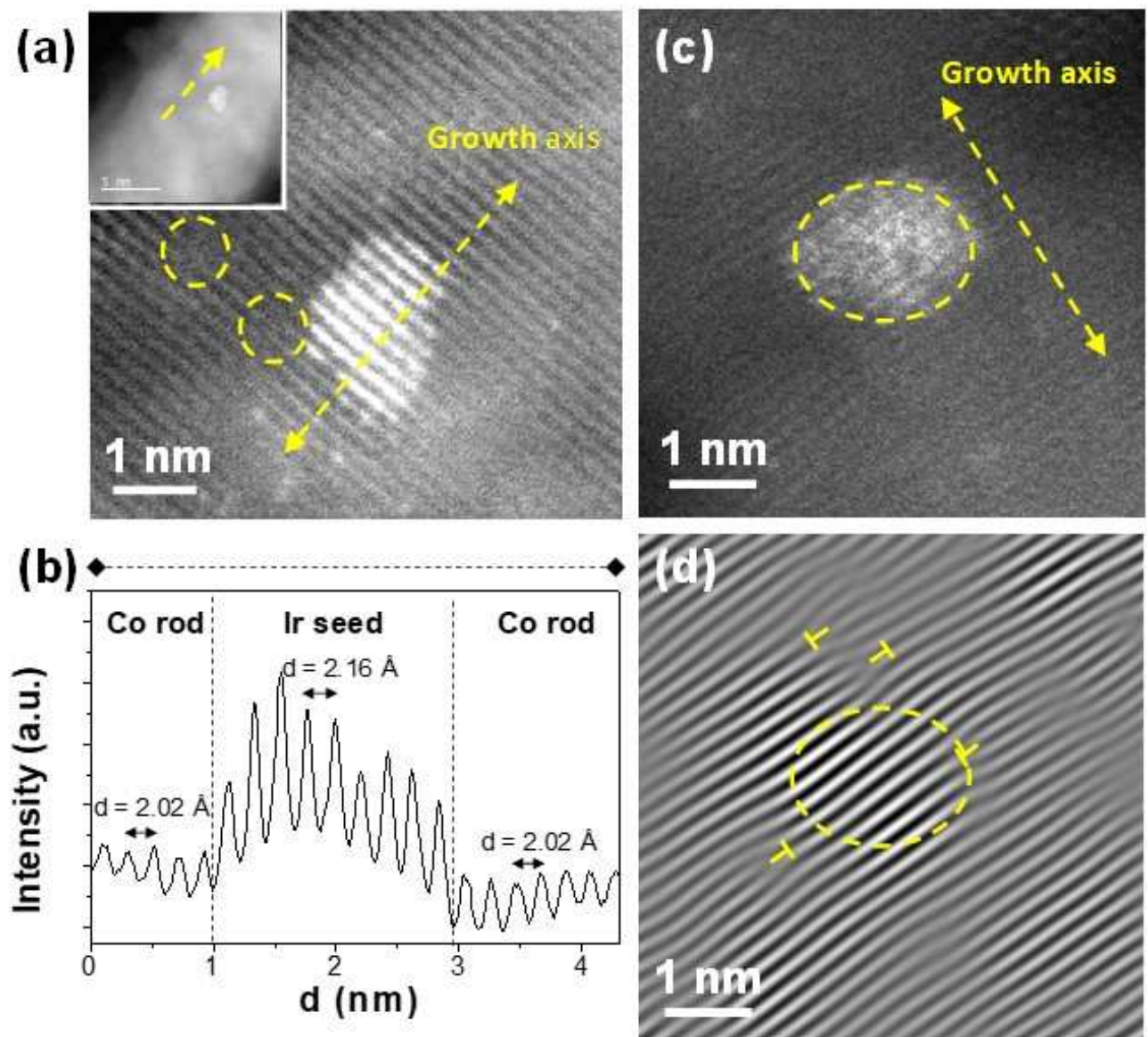

Figure 3. (a) High resolution HAADF-STEM image of an Ir seed in a Co rod, the inset shows an enlarged view of the rod, the yellow circles are guide for the eyes showing the emergence of edge dislocations; (b) Interplanar spacing measured along the yellow line crossing the Ir seed in the figure $3 a$, the distance in the cobalt rod is $2.02 \AA$ corresponding to the (0002) planes and is $2.16 \AA$ inside the seed corresponding to an average value of the (0002) planes of hcp Co and the (111) planes of $\mathrm{fcc} / \mathrm{r}$; (c) High resolution HAADFSTEM image of another Ir seed and (d) Fourier filtered image of the (0002) planes showing more clearly the structural defects around the seed.

A representative HR HAADF-STEM image of a $\mathrm{CO}_{\mathrm{Ru}}$ nanorod is shown in Figure 4. As mentioned above, such rods crystallize with the hcp structure and are single crystals. No interruption of the $\{10 \overline{1} 0\}$ and $\{0002\}$ lattice planes when crossing the Ru seed can be observed. This is consistent both with Ru seeds that crystalize with the hcp structure, like the pure Ru nanoparticles prepared by the polyol process in absence of cobalt, ${ }^{56}$ and with an epitaxial growth of Co on the seed. Unlike for $\mathrm{CO}_{\mathrm{Ir}}$ rods, no structural defect around the Ru seeds was detected. The strains in the cobalt rod surrounding the Ru seeds were determined using the geometric phase analysis (GPA) method. ${ }^{57}$ The GPA method aims to measure the displacement of atomic positions with respect to a perfect 
unstrained lattice. In order to avoid artefacts related to the scanning mode imaging, two sets of 15 fast HR HAADF-STEM images of the rod were recorded in two perpendicular orientations. The images were then precisely aligned with a sub-pixel precision by cross-correlation method and summed (see experimental details). The (0002) and (0002) spots in the Fourier transform of the HR image were selected for the GPA analysis given on Figure 4c. The reference area was chosen in the Co part far from the seed. The Ru seed is revealed by a region of larger lattice parameter, called zone of expansion. On top of the seed the (0002) interplanar spacing is $4 \%$ larger than for pure $\mathrm{Co}$. This value is compatible with a $c$ parameter of ruthenium $5.2 \%$ larger than that of cobalt. The GPA analysis revealed two zones of compression in the cobalt located nearby the Ru seed indicating that the ruthenium structure is coherent with the hcp cobalt. The cobalt atoms around the seeds are displaced to "make room " for the larger precipitate of hcp Ru without any dislocations. It is important to note that these compression zones prove unambiguously that the noble metal seed is located inside the Co particle and not deposited onto its external surface. Few Ru NPs were found on the TEM images that are physisorbed NPs on the cobalt particle surface that were not removed by the washing procedure. Around such particles, no microstructural deformation was observed. GPA is thus highly valuable tool to confirm heterogeneous nucleation mechanisms with tiny seeds.

High resolution HAADF-STEM images were also performed on $\mathrm{CO}_{\mathrm{Ru}}$ nanoplatelets. The Fast Fourier transform was indexed as hcp cobalt in the [0001] zone axis (Figure S15) and again the absence of structural defect on top and nearby the ruthenium seeds was noticeable (Figure 4e-f). The [0001] zone axis affords to measure the parameter $a$ of the hcp structure on top of and beside the seed. An expansion of $a$ comprised between 4 and $5 \%$ with respect to pure cobalt was measured on top of the Ru seed. This expansion is in agreement with the larger parameter $a$ of hcp Ru ( $2.7058 \AA$ ) compared to the one of hcp Co (2.5031 $\AA$ ).

According to the high magnification images, it can be concluded to an epitaxial growth of cobalt both on the Ir and Ru seeds. In the Colr rods, the lattice mismatch accommodation occurs through the formation of dislocations in the immediate vicinity of the seeds while in the $\mathrm{CO}_{\mathrm{Ru}}$ rods and platelets no dislocation was detected and the relaxation occurs through compression zones in the cobalt lattice nearby the seeds. The formation of dislocations at the interface of two metals is generally attributed to a strong lattice mismatch between the metals. The misfits between the Ir (111) and Co (0002) interplanar spacings and between the Ru (0002) and Co (0002) interplanar spacings are $8.9 \%$ and $5.6 \%$, respectively. The lower mismatch between the cobalt and ruthenium parameters compared to iridium could be an explanation for the relaxation of the interatomic distances without misfit dislocations. Nevertheless, the contrast is much weaker if we consider the interatomic distances in the Co (0002), Ru (0002) and $\operatorname{Ir}(111)$ planes. The misfit is $7.5 \%$ between $d_{R u-R u}(2.7058 \AA)$ and $d_{\text {Co-Co }}(2.503 \AA)$ and is $7.8 \%$ between $d_{\text {Ir-lr }}(2.715 \AA)$ and $d_{\text {Co- }}$ co. One hypothesis to explain the absence of dislocation in the $\mathrm{CO}_{\mathrm{Ru}}$ nanoparticles could be the formation of a Co-Ru alloy at the interface between the Ru seed and the Co rod. 
The presence of defects around the Ir seeds in the Colr nanoparticles can have strong effects on their physico-chemical properties such as the morphology change of the Colr rods reported after catalytic tests of alcohol dehydrogenation. ${ }^{44}$ It was observed that the Colr rods mean length decreased by a factor of 2 after being heated at $453 \mathrm{~K}$ during $24 \mathrm{~h}$ in octan-2-ol, and was almost constant after subsequent tests in the same conditions. The mean diameter, on the other hand, remained constant during the repeated tests (see more detail in supplementary information, Figure S16). The decrease of the rod mean length is well explained by a selective chemical etching at the middle of the rods exactly where the seeds are located. The defects around the seeds may act as preferential site for the chemical oxidation of the rods. It is noticeable that once the mean length decreased by a factor of 2 , no further decreasing was observed after subsequent catalytic tests, again in agreement with a selective oxidation at the seeds. 

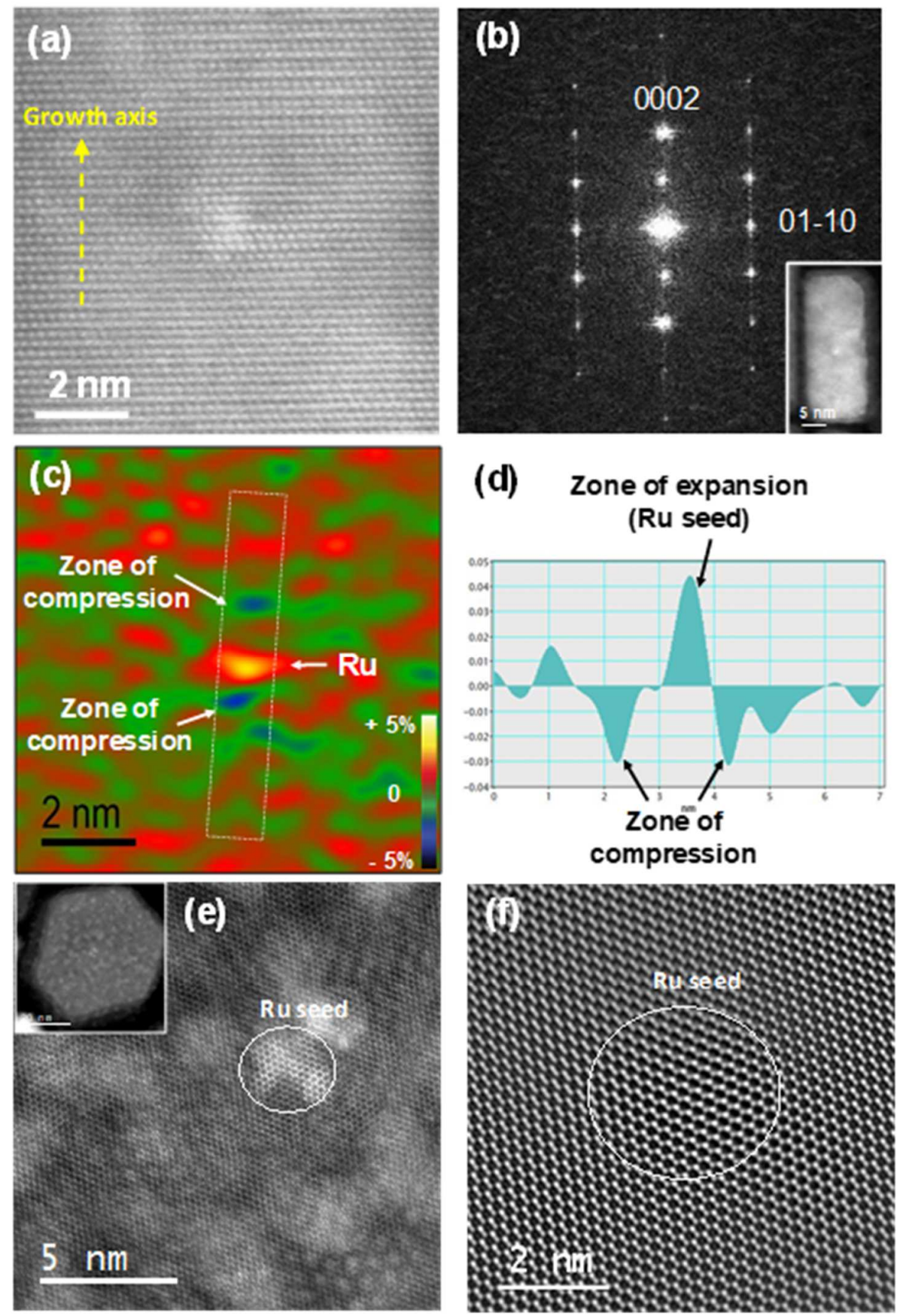

Figure 4. (a) High resolution HAADF-STEM image of a Ru seed in a Co rod; (b) Fast Fourier transform of the HAADF-STEM image. Inset: HAADF-STEM image of the rod; (c) Deformation map of the (0002) planes obtained by the GPA method applied on the HAADF image; (d) distance of the (0002) lattice fringes around the Ru seed showing a zone of lattice parameter expansion on the seed and two zones of compression nearby the seed; (e) High resolution HAADF-STEM image of a Ru seed in a Co platelet viewed along the [0001] zone axis; (f) Fourier filtered image showing structural coherence between the seed and the cobalt. 
In order to have a deeper insight into the nucleation step, an in situ probe of the noble metal seed nucleation and cobalt growth was seeked. Recent studies on the catalytic properties of Co NPs towards the dehydrogenation of alcohols showed that unsupported Co NPs are efficient catalysts for the dehydrogenation of long-chain model secondary mono-alcohols into the corresponding ketones with the concomitant production of pure molecular hydrogen ${ }^{44,48}$ following an acceptorless mechanism. ${ }^{58}$ It was also reported that during the synthesis of hcp Co NRs in 1,2 butanediol, the secondary alcohol function of the polyol was selectively oxidized into a ketone with the formation of molecular hydrogen. ${ }^{51}$ This was an incentive to investigate the production of molecular hydrogen as a probe for the successive steps involved in the polyol synthesis of Co particles. $\mathrm{H}_{2}$ evolution was measured using a flowmeter connected to the condenser outlet, the gas was analyzed by gas chromatography (see experimental section and Figure S17 of supplementary information for the set-up description).

The reduction of $\mathrm{RuCl}_{3}$ and $\mathrm{IrCl}_{3}$ were studied, first, in the absence of cobalt, in the experimental conditions giving the cobalt rods or platelets. TEM images of $\mathrm{Ru}$ and $\mathrm{Ir}$ particles prepared in different conditions are reported in supplementary information (Figure S18). The reduction of ruthenium (resp. iridium) chloride leads to Ru (resp. Ir) NPs with a mean size between 1 and 2 $\mathrm{nm}$, very similar to the size of the seeds in the cobalt particles. The $\mathrm{H}_{2}$ flow recorded during the reduction of $\mathrm{RuCl}_{3} . \mathrm{xH}_{2} \mathrm{O}$ is given in Figure 5a. A first $\mathrm{H}_{2}$ evolution peak is observed at c.a. $150{ }^{\circ} \mathrm{C}$, concomitantly with the color change of the solution that turned from pale green to brown. It suggests that the reduction of the ruthenium complexes in basic solution of polyol involves, first, the formation of molecular ruthenium hydrides by $\beta$-elimination of ruthenium alkoxide. ${ }^{58}$ These hydrides are good catalysts for the acceptorless dehydrogenation of alcohols with molecular hydrogen production, particularly in basic medium. ${ }^{59}$ Without stabilizing ligands, the Ru hydrides are very unstable. The Ru nanoparticles nucleation results from the decomposition of these hydrides forming ruthenium clusters that releases also molecular dihydrogen. Therefore, the $\mathrm{H}_{2}$ evolution peak is a good in situ probe for precisely defining the nucleation temperature of the $\mathrm{Ru}$ nanoparticles. At $175^{\circ} \mathrm{C}$, a second peak followed by a steady flow of $\mathrm{H}_{2}$ is observed (Figure $5 \mathrm{a}$ ). The $\mathrm{H}_{2}$ production at the plateau temperature results from the oxidative dehydrogenation of the polyol catalyzed by the ruthenium particles. ${ }^{60}$ This reaction produces 1-hydroxy butan-2-one (resp. 1-hydroxy propan-2-one) from the 1,2-butanediol (resp. 1,2-propanediol) and molecular hydrogen. ${ }^{51}$ The shape for the $\mathrm{H}_{2}$ evolution curve observed during the reduction of $\mathrm{IrCl}_{3}$ was similar but the first peak appeared at lower temperature and was much broader while the total $\mathrm{H}_{2}$ production was weaker (Figure $\mathrm{S} 19$, supplementary information).

The $\mathrm{H}_{2}$ evolution was then followed during the one-pot SMG synthesis of cobalt particles with $\mathrm{Ru}$ or Ir seeds. At the temperature at which the noble metal is reduced, a $\mathrm{H}_{2}$ evolution peak was observed (Figure 5b). In the presence of the cobalt precursor, the very dark color of the suspensions prevents from observing any color change associated with the reduction of the metal 
ions showing the interest of the $\mathrm{H}_{2}$ evolution as a nucleation probe. The first peak is followed by a strong second $\mathrm{H}_{2}$ evolution peak at $175{ }^{\circ} \mathrm{C}$, indicative of the cobalt particle growth immediately followed by the catalytic activity of the cobalt particles for the oxidative dehydrogenation of the polyol. After the cobalt growth, the $\mathrm{H}_{2}$ production rate is almost constant. This rate is much higher than with the noble metal seeds alone because the metal concentration is 40 times higher.
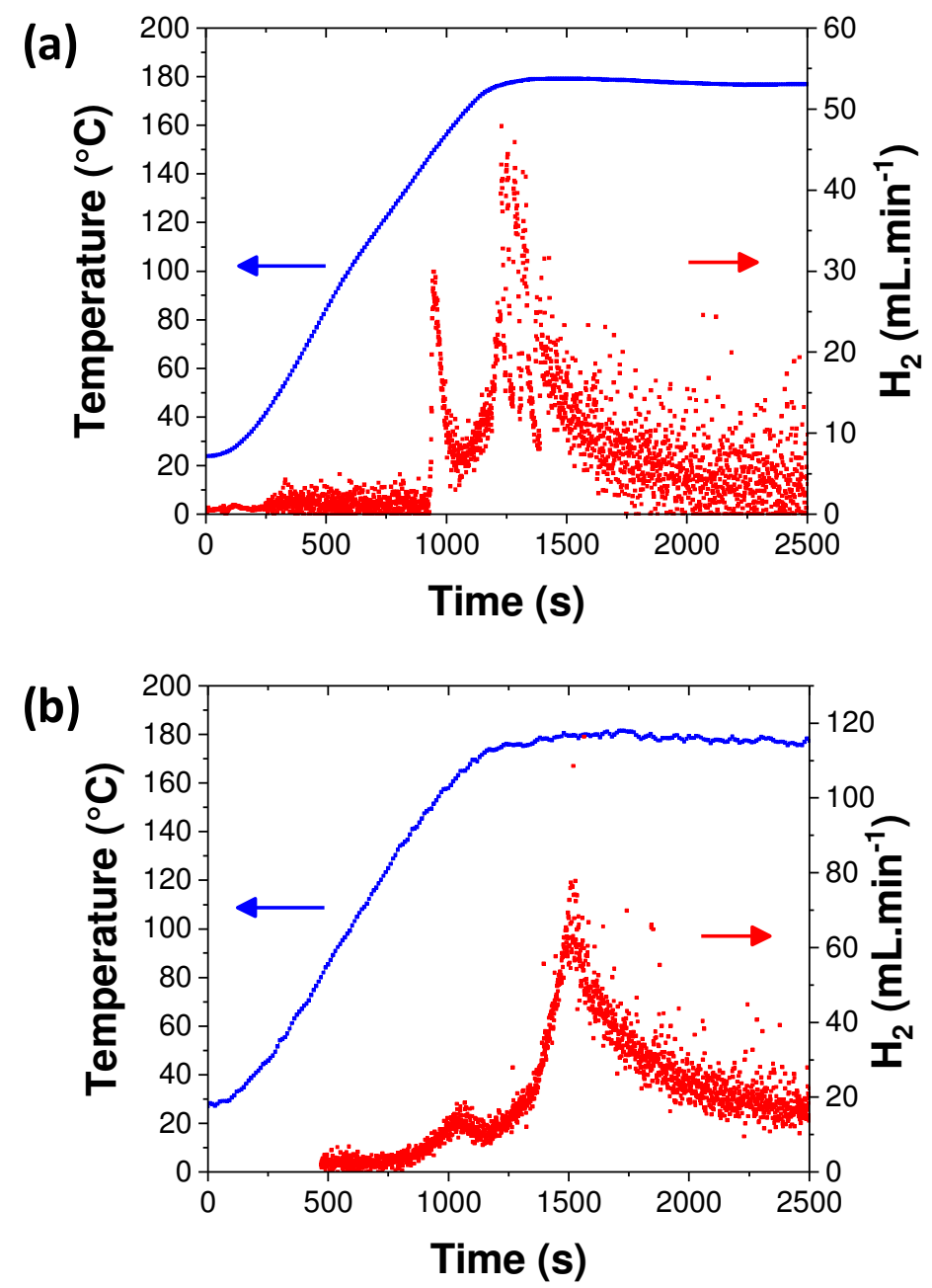

Figure 5. Reaction temperature and $\mathrm{H}_{2}$ flow as function of time measured during the reduction of $2 \mathrm{mM}$ hydrated ruthenium chloride in a solution of $\mathrm{NaOH}$ in 1,2-butanediol (a) and during the reduction of 80 $\mathrm{mM}$ of cobalt dodecanoate in a solution of $\mathrm{NaOH}$ in 1,2-butanediol in presence of $2 \mathrm{mM}$ hydrated ruthenium chloride added to seed the medium (b).

A general scheme for cobalt particle formation can be drawn including, first, the nucleation of the noble metal followed by the symmetric growth of the cobalt from the noble metal seeds (Scheme 1). Due to their higher redox potential, the temperatures of reduction of $\operatorname{Ir}(\mathrm{III})$ and $\mathrm{Ru}$ (III) are 
lower than that of $\mathrm{Co}(\mathrm{II})$. The in situ formation of ultrasmall iridium and ruthenium particles (nucleation step) is concomitant with $\mathrm{H}_{2}$ evolution and followed by the reduction of cobalt at the surface of the seeds (heterogeneous nucleation) and by the cobalt growth, this last step being controlled by the long-chain carboxylate concentration and the nature of the polyol. The observation of two well-separated $\mathrm{H}_{2}$ evolution peaks reveals a good separation of the nucleation and growth steps. No cobalt particles were observed that did not contain a noble metal seed in the center showing that all the cobalt particles were generated through a heterogeneous nucleation. The cobalt growth is perfectly symmetrical from the seeds explaining that the seeds are found exactly in the center of the particles, whatever the cobalt faces favored during the growth and the final particle shapes. According to the phase diagrams, Co is miscible in all proportions with Ir and Ru. ${ }^{61}$ Nevertheless, the cobalt growth is very fast and is carried out at relatively low temperature limiting the inter-diffusion of cobalt atoms and the seeds. In case of a strong inter-diffusion process, a poorer contrast on the HAADF-STEM images would have been observed. In the case of the Corr particles, the presence of dislocations very close to the Ir seeds, due to a strong lattice mismatch, argues also in favor of the absence of inter-diffusion. In the case of the $\mathrm{CO}_{\mathrm{Ru}}$ particles, the formation of a Co-Ru alloy at the interface that could relax the lattice mismatch cannot be completely discarded. An atomic inter-diffusion process could account for the irregular shapes of some seeds observed in the platelets. A slight overlap of the ruthenium and cobalt reduction steps could also pleads in favor of a Ru-Co composition gradient at the surface of the Ru seeds.

The molecular hydrogen evolution concomitant with the seed nucleation strongly supports the formation of metal hydrides as intermediate species resulting from $\beta$-elimination of metal alkoxides. This pathway may explain the different behavior of $\mathrm{RuCl}_{3} \cdot \mathrm{xH}_{2} \mathrm{O}$ and anhydrous $\mathrm{RuCl}_{3}$ as nucleating agent. The presence of water and/or hydroxyl group in the $\mathrm{Ru}$ (III) coordination sphere of the hydrated compound may change the reactivity toward the polyol dehydrogenation. This subtle change in the coordination chemistry could explain a higher reactivity of the anhydrous ruthenium chloride and the formation of a higher number of seeds at lower temperature leading to smaller cobalt particles. 


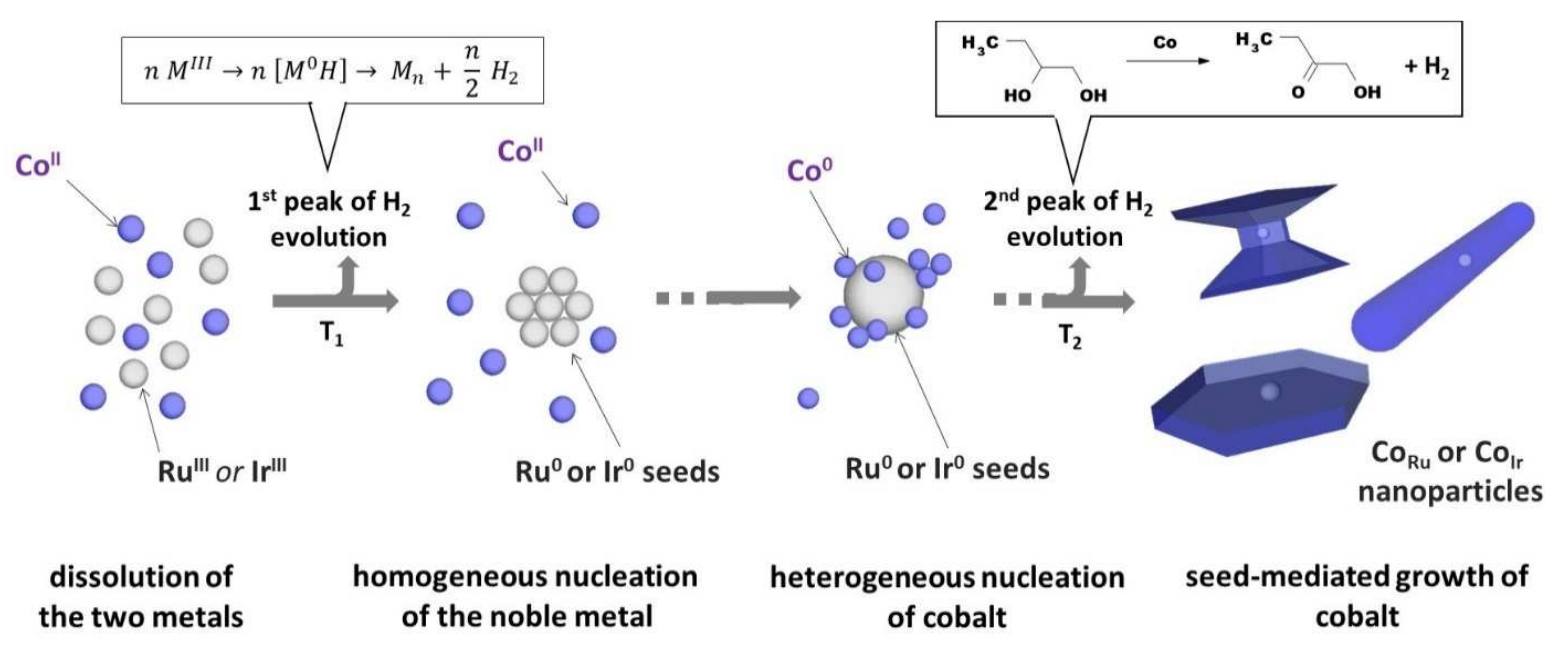

Scheme 1. Different steps and chemical reactions involved in the formation of the one pot in situ seed mediated growth of cobalt nanoparticles in 1,2-butanediol and 1,2-propanediol.

\section{CONCLUSION}

The presence of the noble metal seeds inside cobalt particles prepared by a seed-mediated polyol method has been evidenced for the first time. Whatever the Co particle shape, which is related to the growth mechanism, the seeds are exactly located in the center of the particles in agreement with a one-step seed mediated growth process. Thanks to in situ measurements of molecular hydrogen evolution, the separation of the nucleation and growth steps was clearly established with, first, the nucleation of the noble metal seeds and, at higher temperature, the heterogenous nucleation and growth of cobalt. The classical theory of heterogeneous nucleation states that the noble metal seeds decrease the activation energy barrier for the Co nucleation at their surface. As far as the formation of hydrides and $\mathrm{H}_{2}$ at the surface of the seeds is suspected, one question is still open: could $\mathrm{H}_{2}$ on the surface of the seeds help the reduction of the cobalt precursor, in other words, would there be an additional catalytic effect provided by noble metal seeds? This could be the subject of interesting additional study.

The seeded materials discussed herein are distinct from core-shell materials in that there is a very large difference between the seed size and the final particle size, the molar and volume ratio between the seed and the particle are less than $1 \%$ and, at first approximation, the presence of the seed should not influence the properties of the final material. It might be interesting in a future study to investigate the effect of the seed on the magnetic properties of the cobalt rods. On one hand, the presence of defects around the seeds could locally modify the magnetocrystalline anisotropy of the cobalt and favors the magnetization reversal, but in the other hand, 
since the magnetization reversal nucleates at the tips, ${ }^{35}$ it is believed that structural defects located in the middle of the rods does not affect the coercivity values. The presence of extended defects around the Ir seeds can explain however why the Colr nanorods are more sensitive to oxidation than the $\mathrm{CO}_{\mathrm{Ru}}$ nanorods. The defects located around the seeds may act as preferential site for chemical oxidation. Another perspective to this work will be to clarify why the accommodation of the lattice mismatch between the two kinds of seeds (Ir and Ru) and the hcp Co is so different. The smaller difference of the lattice mismatch between Ru and Co and the fact that they crystallize in the same structure is a good explanation but chemical effects cannot be discarded. One hypothesis is the formation of a Ru-Co alloy at the surface of the seed favored by a higher reduction temperature of Ru compared to Ir and/or by higher production of molecular hydrogen with the ruthenium seeds that could change the surface chemistry of the seeds.

\section{EXPERIMENTAL SECTION}

\section{Synthesis}

Cobalt acetate hydrate, $\mathrm{Co}\left(\mathrm{CH}_{3} \mathrm{COO}\right)_{2} \cdot 4 \mathrm{H}_{2} \mathrm{O}$, (Sigma Aldrich, $\geq 98 \%$ ), dodecanoic acid (Alfa Aesar, 98.5\%), palmitic acid (Sigma Aldrich, $98 \%$ ), $\mathrm{NaOH}$ (Acros), $\mathrm{RuCl}_{3}$ (Sigma Aldrich), $\mathrm{RuCl}_{3} \cdot \mathrm{xH}_{2} \mathrm{O}$ (Sigma Aldrich, 99.98\%), $\mathrm{IrCl}_{3} \cdot \mathrm{xH}_{2} \mathrm{O}$ (Alfa Aesar, 99.8\%), 1,2-butanediol (Sigma Aldrich), sodium dodecanoate, $\mathrm{Na}\left(\mathrm{C}_{11} \mathrm{H}_{23} \mathrm{COO}\right)$ (Aldrich, $\geq 99 \%$ ) and 1,2 propanediol (Acros) were purchased and used without any further purification. Cobalt dodecanoate and cobalt palmitate were prepared first and used as precursor of the cobalt particles. The detailed protocol for the synthesis cobalt dodecanoate and cobalt palmitate can be found elsewhere. ${ }^{41}$

Colr nanorods (CoIr NRs).

Cobalt dodecanoate $(80 \mathrm{mM}), \mathrm{IrCl}_{3} \cdot \mathrm{xH}_{2} \mathrm{O}$ (molar ratio $[\mathrm{Ir}] /[\mathrm{Co}]=2.5 \%$ ) and $\mathrm{NaOH}(75 \mathrm{mM}$ ) were dissolved in 1,2 butanediol at room temperature. The mixture was stirred mechanically at $80 \mathrm{rpm}$ and heated to $175^{\circ} \mathrm{C}$ with a heating rate of $8{ }^{\circ} \mathrm{C} / \mathrm{min}$. The solution was left under stirring for 30 minutes to ensure complete reduction of the Co precursor. Finally, the NPs suspension was cooled down to room temperature and the particles were recovered by centrifugation ( $8500 \mathrm{rpm}$ for 15 $\mathrm{min}$ ) and washed three times with absolute ethanol.

CORu nanorods $\left(\mathrm{CO}_{\mathrm{Ru}} \mathrm{NRS}\right)$.

Cobalt NRs were prepared with hydrated $\left(\mathrm{RuCl}_{3} \cdot \mathrm{xH}_{2} \mathrm{O}\right)$ or anhydrous ruthenium chloride as nucleating agent using a protocol similar to the one described above. $\mathrm{NaOH}$ (final concentration $55 \mathrm{mM}$ ) was dissolved in 1,2 butanediol at $80^{\circ} \mathrm{C}$. Cobalt dodecanoate $(80 \mathrm{mM})$ and anhydrous or hydrated $\mathrm{RuCl}_{3}$ were added to the basic solution at room temperature (molar ratio $[\mathrm{Ru}] /[\mathrm{Co}]=$ 
$2.5 \%)$. The suspension was stirred mechanically at $80 \mathrm{rpm}$ and heated at $175^{\circ} \mathrm{C}$ with a ramping rate of $8{ }^{\circ} \mathrm{C} \cdot \mathrm{min}^{-1}$. After $10 \mathrm{~min}$ at $175^{\circ} \mathrm{C}$ the cobalt rod suspension was cooled down to room temperature, the particles recovered by centrifugation ( $8500 \mathrm{rpm}$ for $15 \mathrm{~min}$ ) and washed with absolute ethanol and toluene.

$\mathrm{CO}_{\mathrm{Ru}}$ nanorods were also prepared under solvothermal conditions and using a microwave heating. In this case, cobalt dodecanoate, anhydrous ruthenium chloride and the basic solution of 1,2butanediol were introduced in the high pressure reactor and heated to $215^{\circ} \mathrm{C}$ with a heating rate of $25{ }^{\circ} \mathrm{C} \cdot \mathrm{min}^{-1}$. After $10 \mathrm{~min}$ at $215^{\circ} \mathrm{C}$ the cobalt rod suspension was cooled down to room temperature, the particles were recovered by centrifugation ( $8500 \mathrm{rpm}$ for $15 \mathrm{~min}$ ) and washed with absolute ethanol and toluene.

Co Ru nanohourglasses ( $\mathrm{CO}_{\mathrm{Ru}} \mathrm{NHGS}$ ).

Sodium dodecanoate $(80 \mathrm{mM})$ was dissolved in 1,2 butanediol at $80^{\circ} \mathrm{C}$. Cobalt dodecanoate ( 80 $\mathrm{mM}$ ) and anhydrous ruthenium chloride $\mathrm{RuCl}_{3}$ (molar ratio $[\mathrm{Ru}] /[\mathrm{Co}]=2.5 \%$ ) were added as powders to the solution at $40^{\circ} \mathrm{C}$. The suspension was stirred mechanically at $80 \mathrm{rpm}$ and heated to $175^{\circ} \mathrm{C}$ with a heating rate of $8{ }^{\circ} \mathrm{C} \cdot \mathrm{min}^{-1}$. After $20 \mathrm{~min}$ at $175^{\circ} \mathrm{C}$, the suspension was cooled down to room temperature and the particles were recovered by centrifugation ( $8500 \mathrm{rpm}$ for 15 min) and washed four times with methanol and toluene to remove the excess of sodium dodecanoate. Similar synthesis was carried out by reducing cobalt palmitate in a sodium palmitate solution in 1,2-butanediol following the same procedure.

$\mathrm{CO}_{R u}$ nanoplatelets ( $\mathrm{CO}_{\mathrm{Ru}} \mathrm{NPIS}$ ).

Sodium dodecanoate $(160 \mathrm{mM})$ was dissolved in 1,2 propanediol $(100 \mathrm{ml})$ at $80^{\circ} \mathrm{C}$. The mixture was cooled down to $40{ }^{\circ} \mathrm{C}$ before adding cobalt dodecanoate $(80 \mathrm{mM})$, anhydrous $\mathrm{RuCl}_{3}$ (molar ratio $[\mathrm{Ru}] /[\mathrm{Co}]=2.5 \%$ ). The suspension was stirred mechanically at $80 \mathrm{rpm}$ and heated at the rate of $8{ }^{\circ} \mathrm{C} \cdot \mathrm{min}^{-1}$, from room temperature to $175^{\circ} \mathrm{C}$. After $20 \mathrm{~min}$ the suspension was cooled down to room temperature and the Co nanoplatelets were collected by centrifugation ( $8500 \mathrm{rpm}$ for $15 \mathrm{~min}$ ) and washed several times with methanol to remove the excess of sodium dodecanoate. Similar synthesis was carried out by reducing cobalt palmitate in a sodium palmitate solution in 1,2-butanediol following the same procedure.

\section{Characterization}

For electron microscope characterizations, the particles were dispersed in toluene and sonicated. The washing procedure favoured the passivation of the cobalt particles by a thin layer of cobalt oxide $\mathrm{CoO}$ with a thickness of c.a. $1.5 \mathrm{~nm}$. Even though the particles have been handled in the air, they have never been oxidized to the core. One drop of the suspension was deposited on a carbon coated copper grid and the solvent was evaporated at room temperature under vacuum. The 
particle morphology was studied with JEOL JEM1011 transmission electron microscope operating at $100 \mathrm{kV}$. The cobalt particle mean sizes were determined on $c a .200$ particles. Besides the cobalt particles, some very small particles with size between 1 and $2 \mathrm{~nm}$ were also observed. These particles are Ir or Ru nanoparticles that were produced by reduction of the corresponding chlorides but that did not serve as seeds for the cobalt growth. Very few of these small particles were found physisorbed at the surface of the cobalt rods. High-resolution electron microscopy and geometric phase analysis (see below) removed any ambiguity about the nature of these particles.

Scanning transmission electron microscopy coupled with high angle annular dark field imaging (HAADF-STEM) observations and local analysis by energy dispersive X-ray spectroscopy (EDS) were performed on a probe-corrected JEOL JEM-ARM200F instrument equipped with a cold field emission gun and operated at $200 \mathrm{kV}$. Stacks of high-resolution HAADF-STEM images with a low exposure time were obtained thanks to a dedicated software. The correction of the spatial drift between images composing the stack was performed using a cross-correlation-based method before summation for obtaining a final high resolution image used for geometric phase analysis (GPA) were done on a probe-corrected scanning TEM FEI Titan Low-Base 60-300 microscope operated at $300 \mathrm{kV}$. The microscope was equipped with a X-FEG ${ }^{\circledR}$ gun and $\mathrm{Cs}$-probe corrector (CESCOR from CEOS $\mathrm{GmbH}$ ).

X-ray diffraction (XRD) pattern were recorded on a PANalytical Empyrean diffractometer in the $2 \theta$ range $10-90^{\circ}$, using CoK $\alpha$ radiation $(\lambda=1.789 / 1.792 \AA)$.

\section{In situ measurements}

The volume of generated $\mathrm{H}_{2}$ was measured as function of time using a Bronkhorst flow meter. A schematic representation of the experimental setup is shown on the Figure S17a of the supplementary information. The nature of the gas collected during the reaction was analyzed by mass spectrometry with a Pfeiffer Omnistar GSD320 apparatus (Figure S17b).

\section{ASSOCIATED CONTENTS}

\section{Supporting information}

Figures showing TEM images, XRD patterns and conventional high-resolution TEM images of $\mathrm{CO}_{\text {Ir }}$ and $\mathrm{CO}_{\mathrm{Ru}}$ nanorods, platelets and hour-glass like particles; additional HAADF-STEM images and local EDS analyses of Colr and $\mathrm{CO}_{\mathrm{Ru}}$ nanorods and platelets; experimental setup monitoring the $\mathrm{H}_{2}$ evolution during the cobalt NPs synthesis and $\mathrm{H}_{2}$ evolution curves; TEM images and size distribution of CoIr nanorods after catalytic tests. 


\section{AUTHORS INFORMATION}

Corresponding Authors

*E-mail: gviau@insa-toulouse.fr ; phone: +33567048833 ; fax: +33561559697.

*E-mail: jean-yves.piquemal@univ-paris-diderot.fr ; phone: +33157278766.

\section{ACKNOWLEDGEMENTS}

This work was funded by the French National Research Agency, ANR, in the framework of the TANOPOL project (ANR-15-CE07-0011-01). Angélique Gillet is warmly thanked for the nanoparticles synthesis, Lucien Datas and Teresa Hungria (Plateforme Castaing, Toulouse) for HAADF-STEM images. We gratefully acknowledge the International Associated Laboratory (LIA)$M^{2}$ OZART for financial support. High resolution HAADF-STEM images were performed at the Laboratorio de Microscopias Avanzadas, Instituto de Nanociencia de Aragon, Universidad de Zaragoza, Spain. R. A. gratefully acknowledges the support from the Spanish Ministry of Economy and Competitiveness (MINECO) through project MAT2016-79776-P (AEF/FEDER. UE). 


\section{Supporting Information}

\section{One-Pot Seed-Mediated Growth of Co Nanoparticles by the Polyol Process: Unraveling the Heterogeneous Nucleation}

Raj Kumar Ramamoorthy, ${ }^{1}$ Arnaud Viola, ${ }^{2}$ Bilel Grindi, ${ }^{1}$ Jennifer Peron, ${ }^{2}$ Christophe Gatel, ${ }^{3}$ Martin Hytch, ${ }^{3}$ Raul Arenal, ${ }^{4,5}$ Lorette Sicard, ${ }^{2}$ Marion Giraud, ${ }^{2}$ Jean-Yves Piquemal, ${ }^{2,}{ }^{*}$ Guillaume $\operatorname{Viau}^{1, *}$

\footnotetext{
${ }^{1}$ Université de Toulouse, UMR 5215 INSA, CNRS, UPS, Laboratoire de Physique et Chimie des NanoObjets, 135 avenue de Rangueil F-31077 Toulouse cedex 4, France

${ }^{2}$ ITODYS, Sorbonne Paris Cité, Université Paris Diderot, 15 rue Jean-Antoine de Baï, 75013 Paris, France

${ }^{3}$ Centre d'Elaboration de Matériaux et d'Etudes Structurales, CEMES-CNRS, 29 rue Jeanne Marvig, B.P. 94347, 31055 Toulouse, France

${ }^{4}$ Laboratorio de microscopias avanzadas (LMA), Instituto de Nanociencia de Aragon (INA), U. Zaragoza, C/ Mariano Esquillor s/n, 50018 Zaragoza, Spain

${ }^{5}$ ARAID Foundation, 50018 Zaragoza, Spain
}

Corresponding Authors

*E-mail: gviau@insa-toulouse.fr ; phone: +33567048833 ; fax: +33561559697.

*E-mail: jean-yves.piquemal @univ-paris-diderot.fr ; phone: +33157278766. 

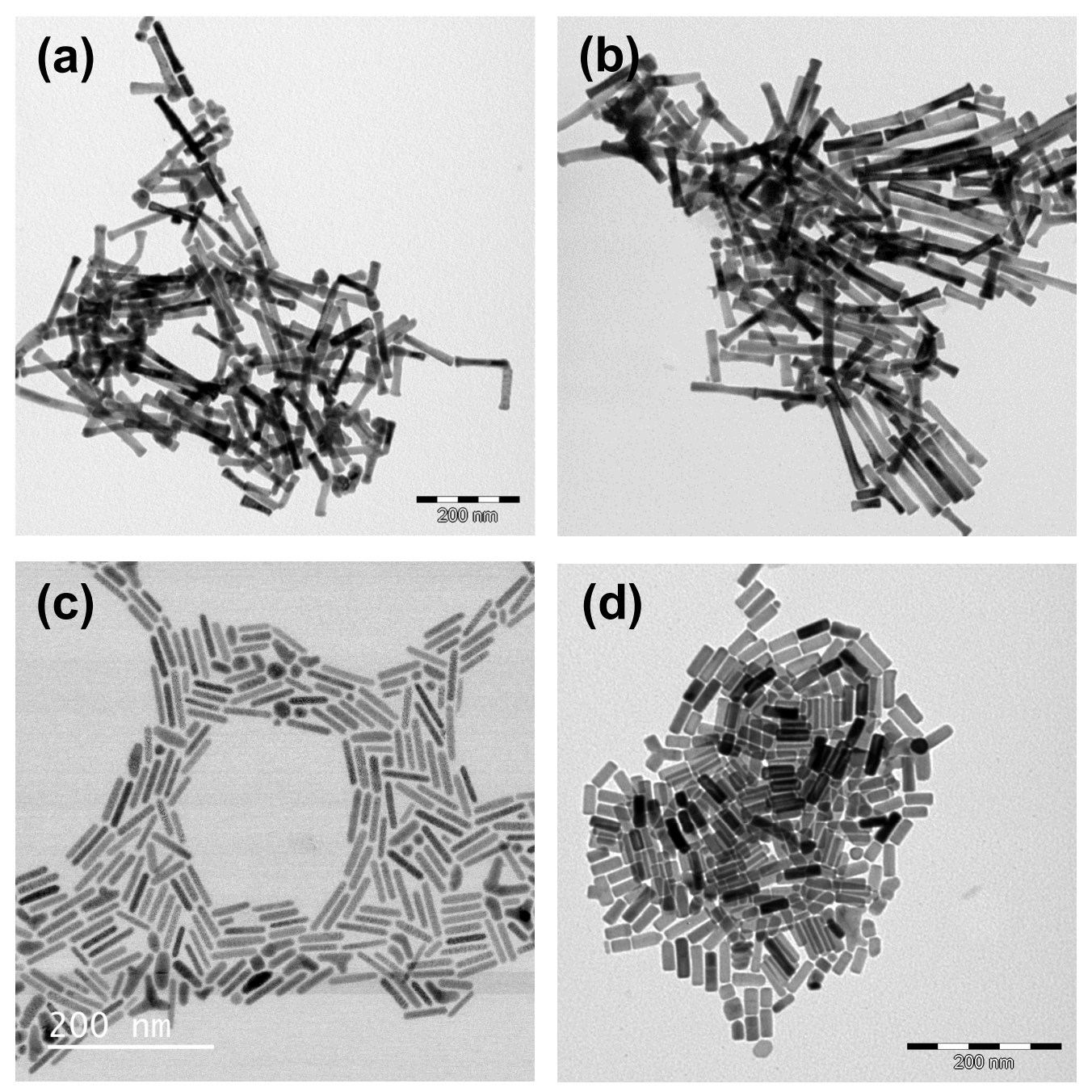

Figure S1. TEM images of cobalt nanorods (NRs) prepared by reducing cobalt dodecanoate (80 $\mathrm{mM}$ ) in a $\mathrm{NaOH}$ solution in 1,2-butanediol using different seeding agent and different heating conditions:

(a) Colr NRs (mean length $L_{m}=105 \mathrm{~nm}$; mean diameter $d_{m}=15 \mathrm{~nm}$ ) obtained with $\operatorname{IrCl}_{3} \cdot \mathrm{xH}_{2} \mathrm{O}$ as seeding agent (molar ratio $\mathrm{Ir} / \mathrm{Co}=2.5 \%$ ) at $175^{\circ} \mathrm{C}$ with a heating rate of $8{ }^{\circ} \mathrm{C} \cdot \mathrm{min}^{-1}$ (classical heating mantle);

(b) $\mathrm{CO}_{\mathrm{Ru}} \mathrm{NRs}$ (mean length $\mathrm{L}_{\mathrm{m}}=125 \mathrm{~nm}$; mean diameter $\mathrm{d}_{\mathrm{m}}=16 \mathrm{~nm}$ ) obtained with $\mathrm{RuCl}_{3} \cdot \mathrm{xH}_{2} \mathrm{O}$ as seeding agent (molar ratio $\mathrm{Ru} / \mathrm{Co}=2.5 \%$ ) at $175^{\circ} \mathrm{C}$ with a heating rate of $8{ }^{\circ} \mathrm{C} \cdot \mathrm{min}^{-1}$ (classical heating mantle);

(c) $\mathrm{CO}_{\mathrm{Ru}} \mathrm{NRs}$ (mean length $\mathrm{L}_{\mathrm{m}}=50 \mathrm{~nm}$; mean diameter $\mathrm{d}_{\mathrm{m}}=10 \mathrm{~nm}$ ) obtained with anhydrous $\mathrm{RuCl}_{3}$ as seeding agent (molar ratio $\mathrm{Ru} / \mathrm{Co}=2.5 \%$ ) at $215^{\circ} \mathrm{C}$ with a heating rate of $25^{\circ} \mathrm{C} \cdot \mathrm{min}^{-1}$ (microwave heating); 
(d) $\mathrm{CO}_{\mathrm{Ru}} \mathrm{NRs}$ (mean length $\mathrm{Lm}_{\mathrm{m}}=45 \mathrm{~nm}$; mean diameter $\mathrm{d}_{\mathrm{m}}=15 \mathrm{~nm}$ ) obtained with anhydrous $\mathrm{RuCl}_{3}$ as seeding agent (molar ratio $\mathrm{Ru} / \mathrm{Co}=2.5 \%$ ) at $175^{\circ} \mathrm{C}$ with a heating rate of $8{ }^{\circ} \mathrm{C} \cdot \mathrm{min}^{-1}$ (classical heating mantle).
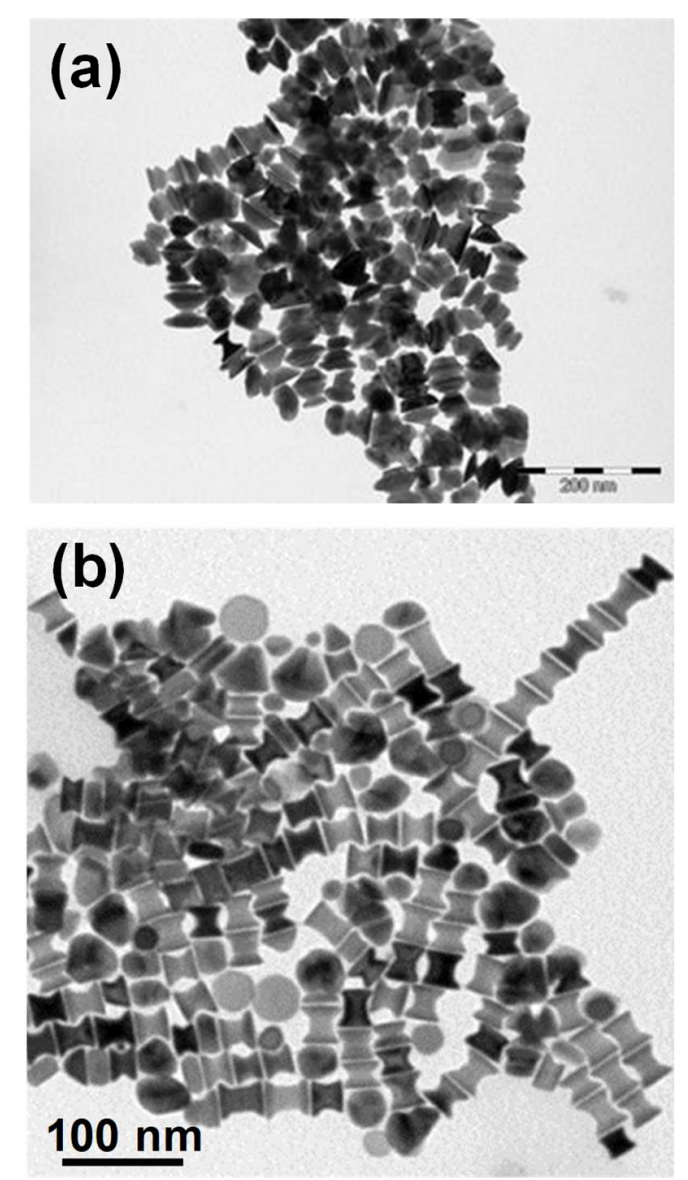

Figure S2. TEM images of cobalt nano-hourglasses (NHGs) prepared by reducing a cobalt carboxylate in a basic solution of 1,2-butanediol, with anhydrous $\mathrm{RuCl}_{3}$ as seeding agent (molar ratio $\mathrm{Ru} / \mathrm{Co}=2.5 \%)$, at $175^{\circ} \mathrm{C}$ with a heating rate of $8{ }^{\circ} \mathrm{C} \cdot \mathrm{min}^{-1}$ :

(a) $\mathrm{Co}_{\mathrm{Ru}} \mathrm{NHGs}$ (mean length $\mathrm{L}_{\mathrm{m}}=39 \mathrm{~nm}$; mean diameter $\mathrm{d}_{\mathrm{m}}$ (center) $=20 \mathrm{~nm}$; mean diameter $D_{m}($ tips $\left.)=45 \mathrm{~nm}\right)$ prepared from cobalt dodecanoate $(80 \mathrm{mM})$ in a solution of sodium dodecanoate $(80 \mathrm{mM})$;

(b) $\mathrm{CO}_{\mathrm{Ru}} \mathrm{NHGs}$ (mean length $\mathrm{L}_{\mathrm{m}}=27 \mathrm{~nm}$; mean diameter $\mathrm{d}_{\mathrm{m}}$ (center) = $17 \mathrm{~nm}$; mean diameter $D_{m}($ tips $\left.)=24 \mathrm{~nm}\right)$ prepared from cobalt palmitate $(80 \mathrm{mM})$ in a solution of sodium palmitate $(80$ $m M)$. 


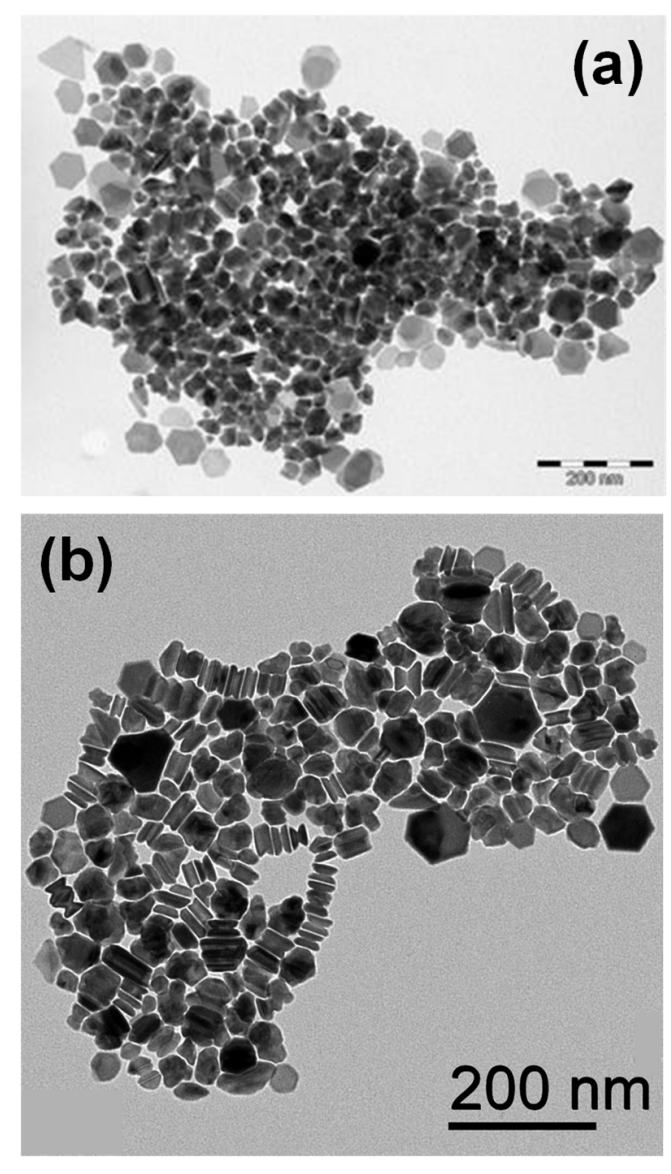

Figure S3. TEM images of cobalt nanoplatelets (NPLS) prepared by reducing a cobalt carboxylate in a basic solution of 1,2-propanediol, with anhydrous $\mathrm{RuCl}_{3}$ as seeding agent (molar ratio $\mathrm{Ru} / \mathrm{Co}=$ $2.5 \%)$, at $175^{\circ} \mathrm{C}$ with a heating rate of $8{ }^{\circ} \mathrm{C} \cdot \mathrm{min}^{-1}$ :

(a) $\mathrm{CO}_{\mathrm{Ru}}$ NPLs (mean thickness $\mathrm{L}_{m}=8 \mathrm{~nm}$; mean diameter $D_{m}=56 \mathrm{~nm}$ ) prepared from cobalt dodecanoate $(80 \mathrm{mM})$ in a solution of sodium dodecanoate $(160 \mathrm{mM})$;

(b) $\mathrm{CO}_{\mathrm{Ru}} \mathrm{NPLs}$ (mean thickness $\mathrm{L}_{m}=12 \mathrm{~nm}$; mean diameter $\mathrm{D}_{\mathrm{m}}=54 \mathrm{~nm}$ ) prepared from cobalt palmitate $(80 \mathrm{mM})$ in a solution of sodium palmitate $(160 \mathrm{mM})$. 


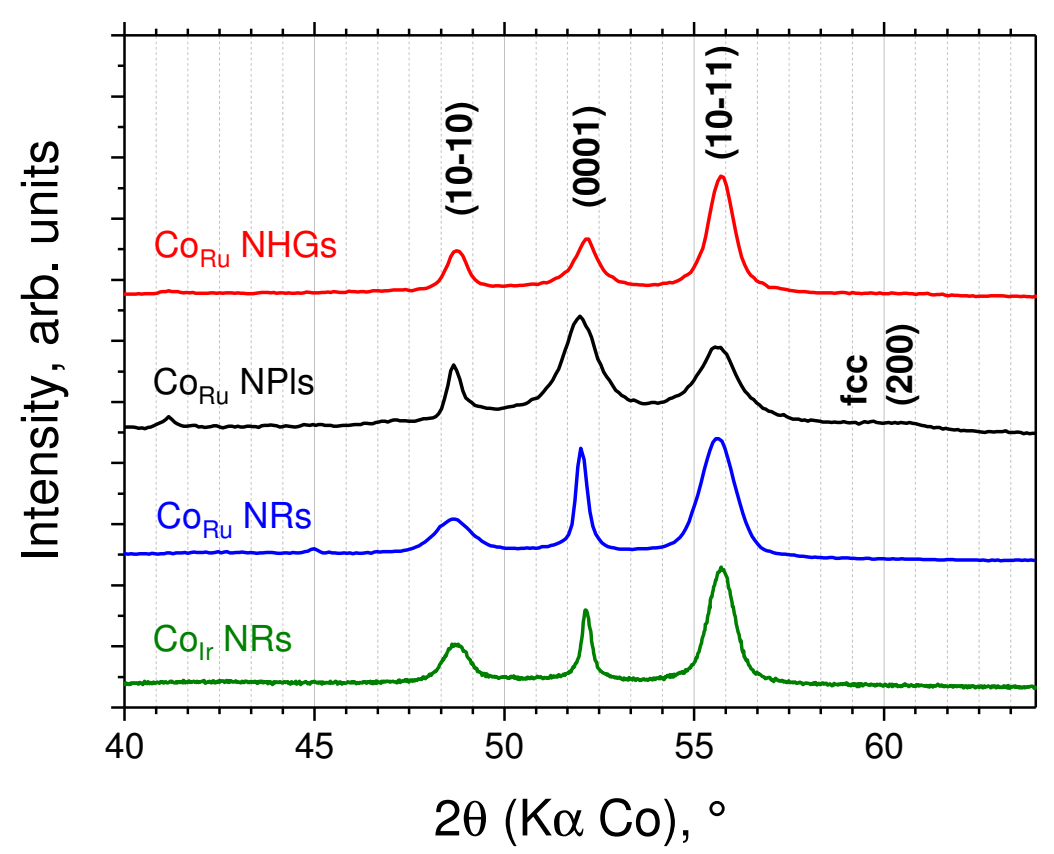

Figure S4. X-ray diffraction patterns of cobalt particles with different morphologies, Co Ir nanorods (COIr NRs), $\mathrm{CO}_{\mathrm{Ru}}$ nanorods ( $\mathrm{CO}_{\mathrm{Ru}}$ NRs with mean length $\mathrm{L}_{\mathrm{m}}=50 \mathrm{~nm}$ and mean diameter $\mathrm{d}_{\mathrm{m}}=10$ $\mathrm{nm}$ ), $\mathrm{CO}_{\mathrm{Ru}}$ nanoplatelets ( $\mathrm{CO}_{\mathrm{Ru}} \mathrm{NPIs}$ ) and $\mathrm{CO}_{\mathrm{Ru}}$ nanohourglasses ( $\mathrm{CO}_{\mathrm{Ru}} \mathrm{NHGs}$ ). The four patterns can be indexed as pure Co hcp phase except the pattern of the platelets that exhibits a small amount of Co fcc. It is noticeable on these pattern that the relative broadening of the $(10 \overline{1} 0)$ and (0002) lines varies from one sample to another and is related to the particle morphology. On the two Co nanorod samples the (0002) lines are much narrower than the (10 $\overline{1} 0)$ lines showing a longer crystallographic coherence along the $c$ axis than perpendicular to it. This is in agreement with a growth of the nanorods along the $c$ axis. In contrast, on the $\mathrm{CO}_{\mathrm{Ru}}$ nanoplatelets pattern the (0002) line appears broader than the (10 $\overline{1} 0)$ line showing a shorter crystallographic coherence along the $c$ axis. This is in agreement with a growth of the nanoplatelets perpendicular to $c$. For the $\mathrm{CO}_{\mathrm{Ru}}$ nanohourglasses the broadening of the (10 $\overline{1} 0)$ and (0002) lines are similar in agreement with particles with an aspect ratio close to 1 . 


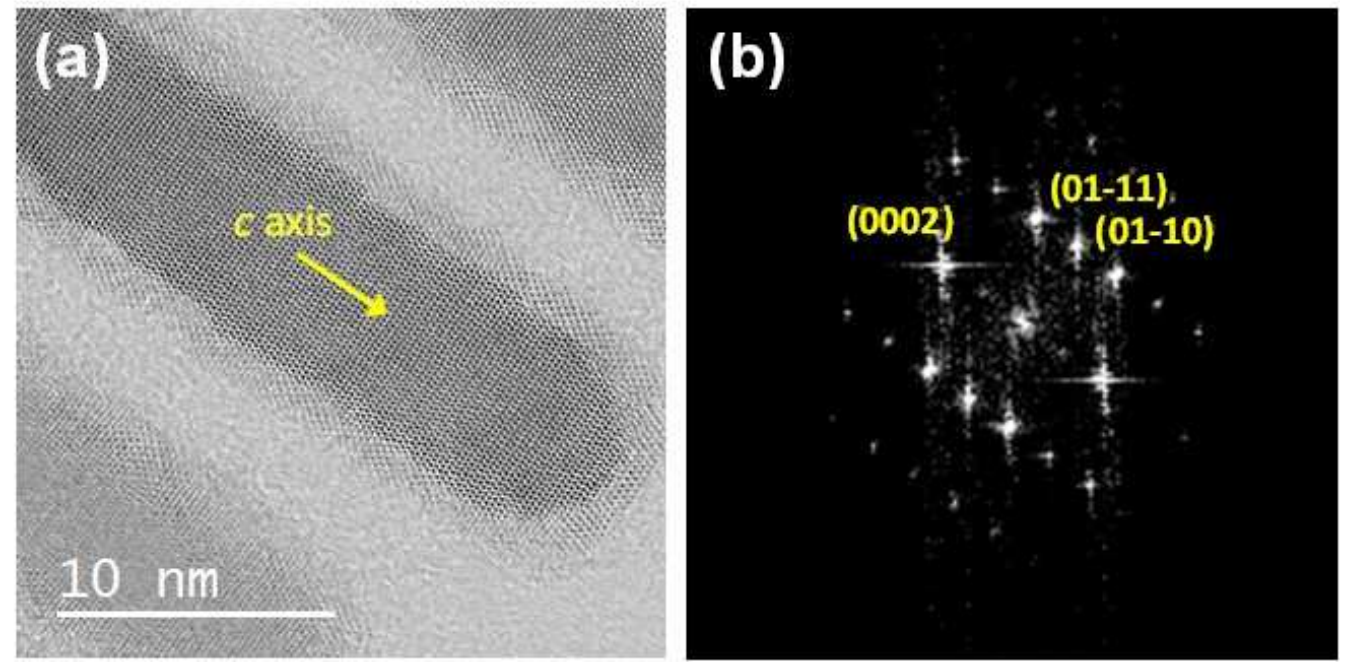

Figure S5. HRTEM image of a $\mathrm{CO}_{\text {Ru }}$ nanorod prepared at $215^{\circ} \mathrm{C}(\mathrm{a})$ and fast Fourier transform (b). The diffraction pattern is indexed as the Co hcp structure in [2 $\overline{1} \overline{1} 0]$ zone axis. The crystallographic axis $c$ is parallel to the long axis. 

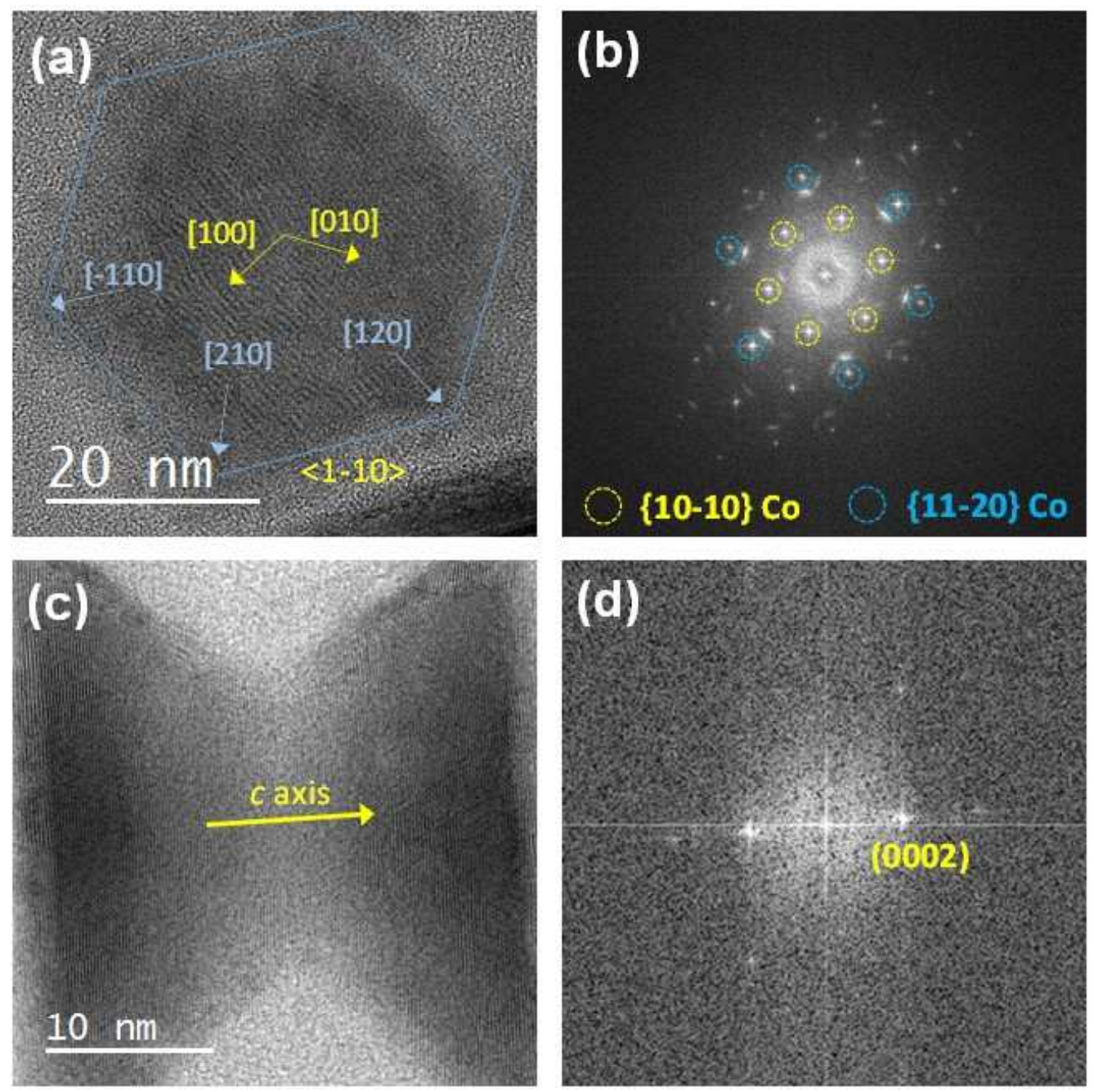

Figure S6. HRTEM image of a $\mathrm{CO}_{\mathrm{Ru}}$ nanohourglass particle viewed parallel to the $c$ axis (a) and fast Fourier transform indexed as a hcp Co particle in [0001] zone axis (b) ; HRTEM image of a CORu nanohourglass viewed perpendicularly to the $c$ axis particle (c) and corresponding viewed parallel fast Fourier transform exhibiting the (0002) reflections. 

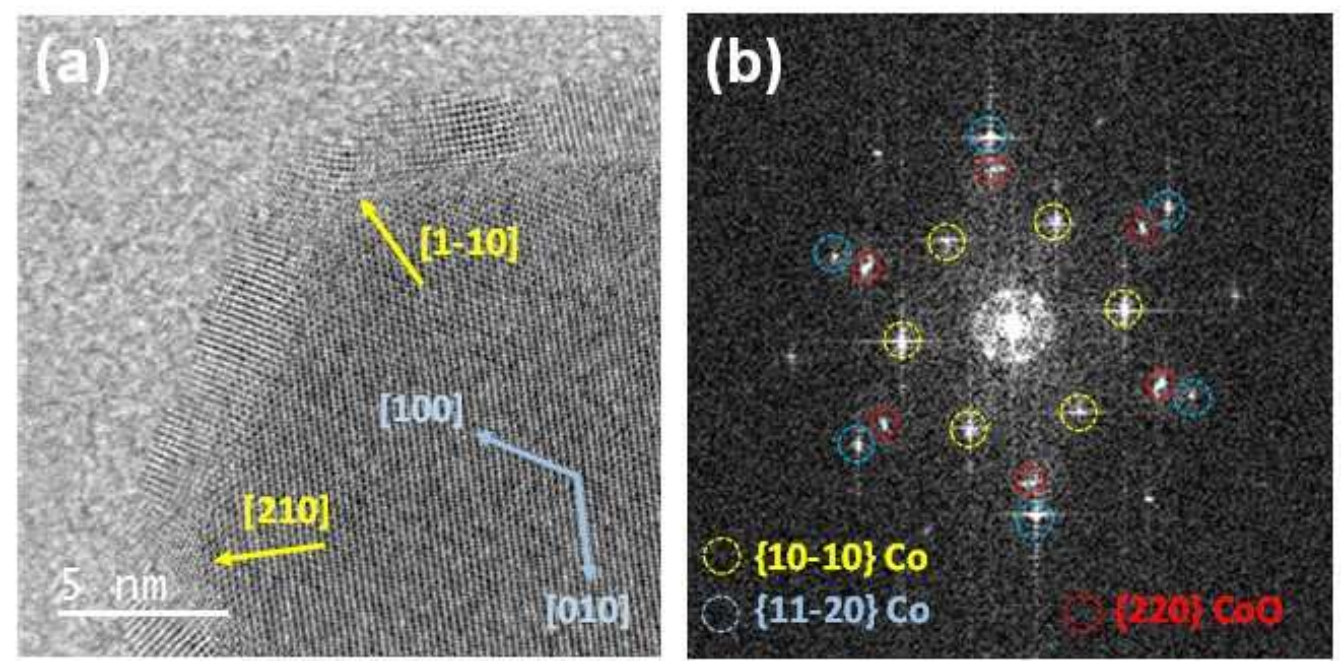

Figure S7. HRTEM image of a $\mathrm{CO}_{\mathrm{Ru}}$ nanoplatelet prepared in 1,2-propanediol (a) and fast Fourier transform (b). The diffraction pattern is indexed as the Co hcp structure in [0001] zone axis. The crystallographic axis $c$ is perpendicular to the platelet. The $\langle 100\rangle$ directions (blue arrows) point towards the edges of the platelet while the $\langle 1 \overline{1} 0\rangle$ directions (yellow arrows) point towards the vertices. 

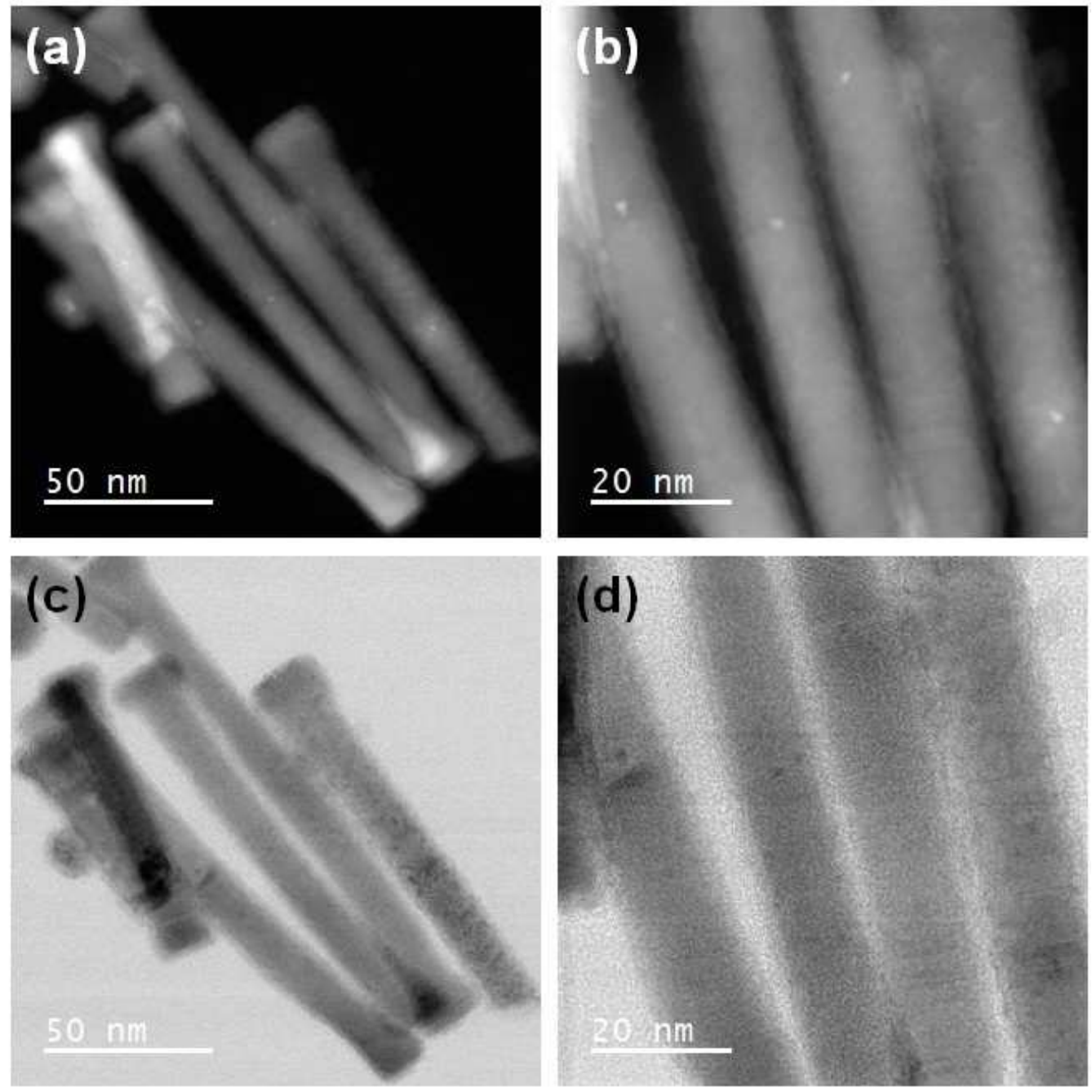

Figure S8. (a) and (b) HAADF-STEM images of cobalt nanorods prepared with $\mathrm{IrCl}_{3}, \mathrm{x} \mathrm{H}_{2} \mathrm{O}$ as nucleating agent ; (c) and (d) corresponding bright field images. 


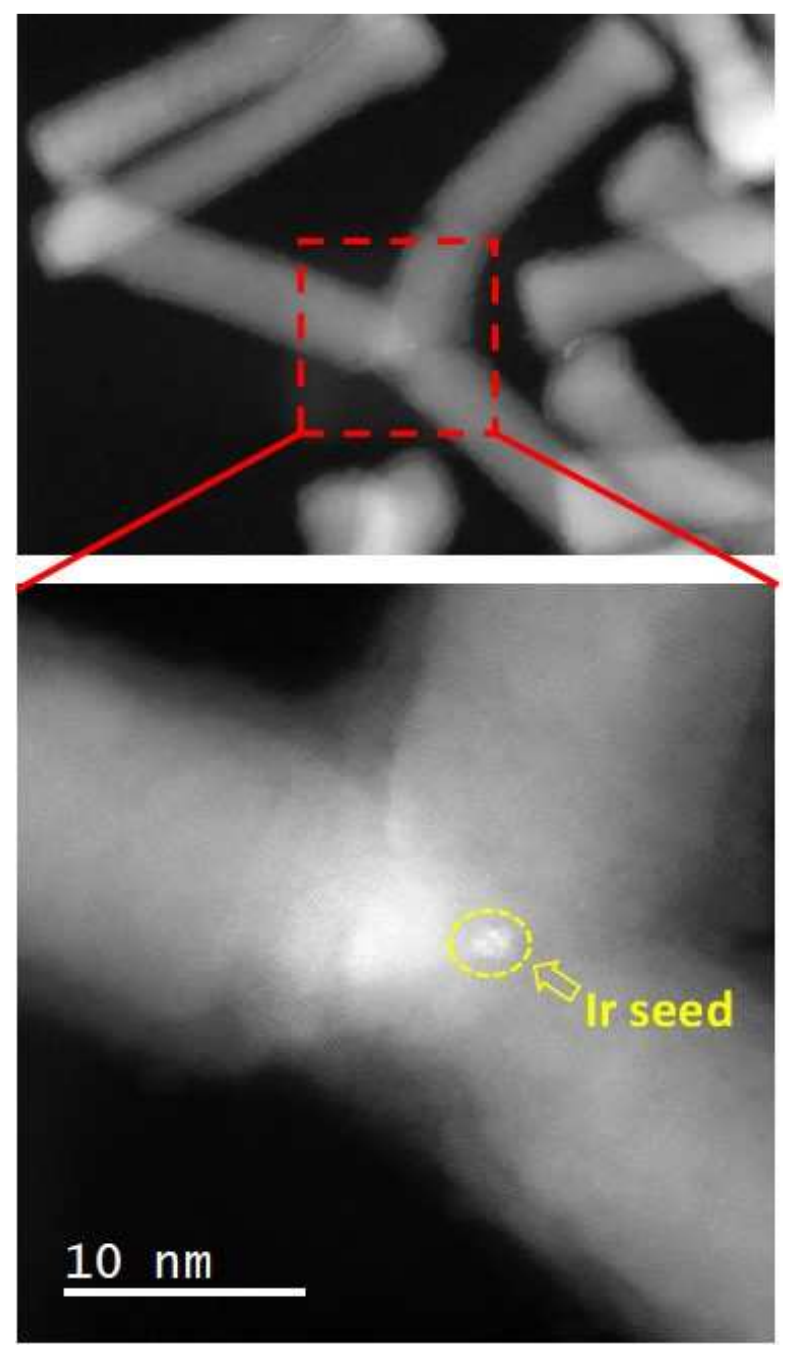

Figure S9. HAADF-STEM image of a cobalt tripod prepared with $\mathrm{IrCl}_{3}, \mathrm{x}_{2} \mathrm{O}$ as nucleating agent; The Ir seed is located at the junction of the three rods. 

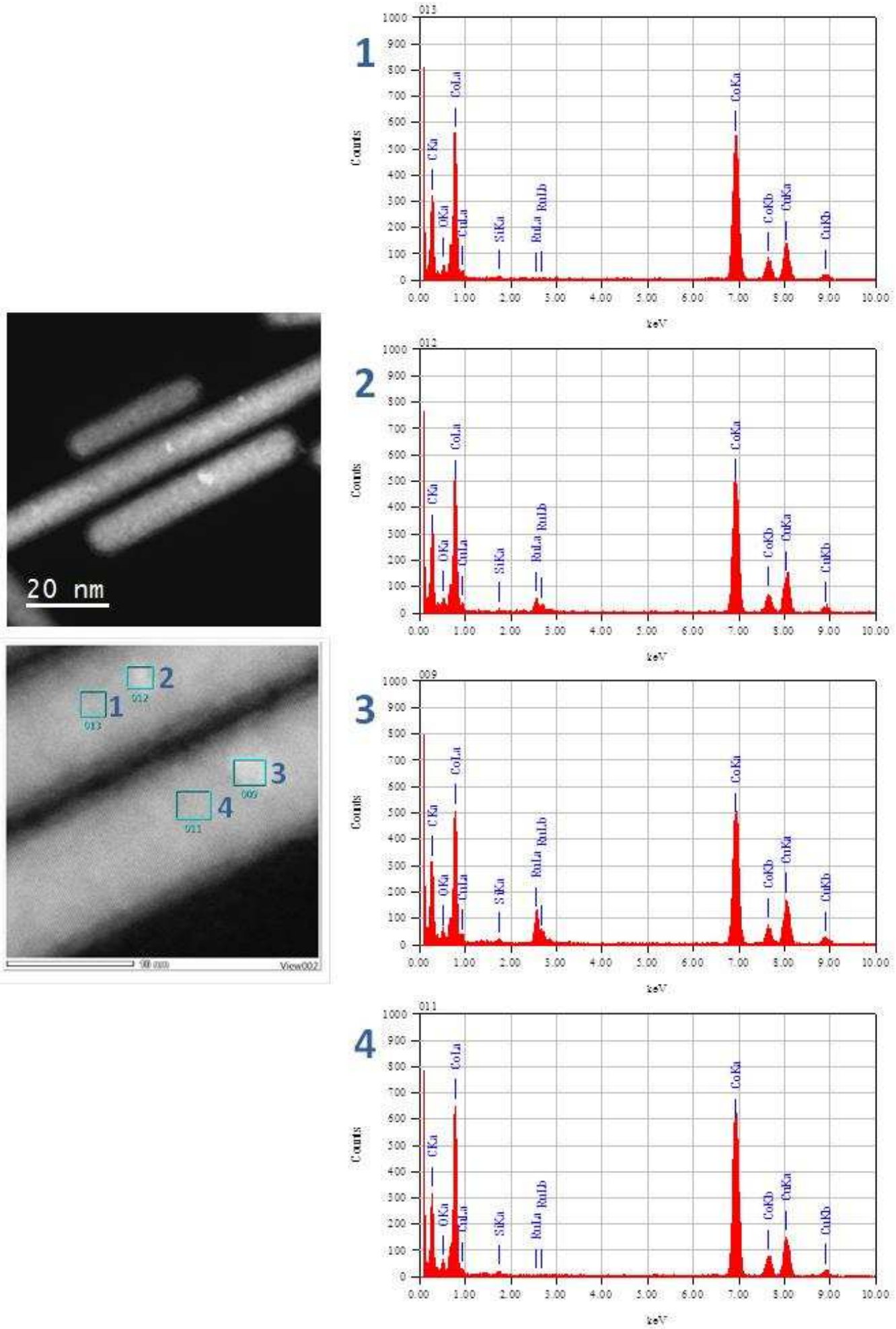

Figure S10. Local Energy dispersive analysis showing that the bright zones of the HAADF-STEM image contain Ru. 


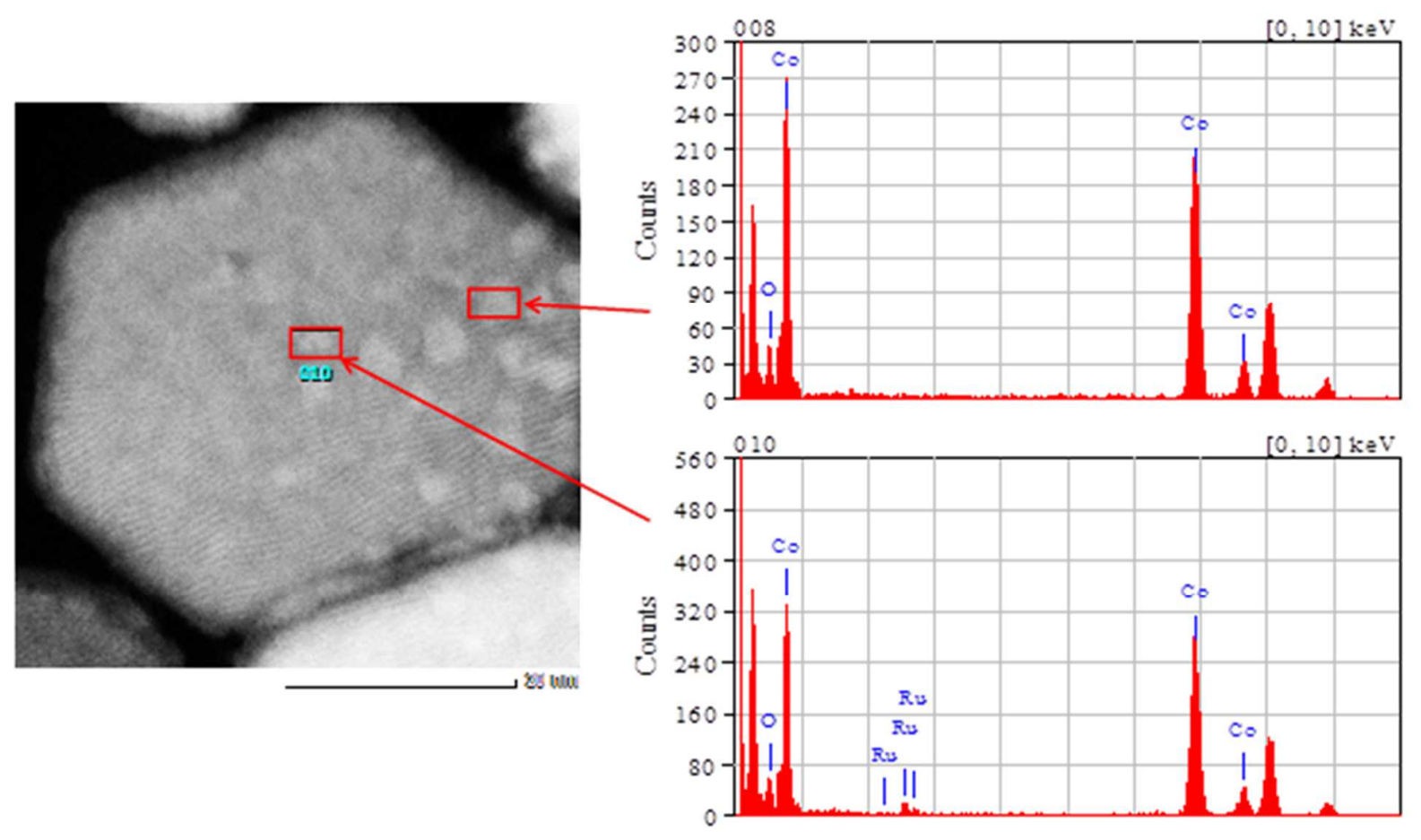

Figure S11. Local Energy dispersive analysis on two bright zones of a cobalt platelet showing that the center zone contains Ru and that the other does not contain any Ru. The Ru seed is single in the platelet and is located exactly in the center of the particle. The other bright zones correspond to thicker area due to the growth of $\mathrm{CoO}$ islands at the surface of the platelet. 


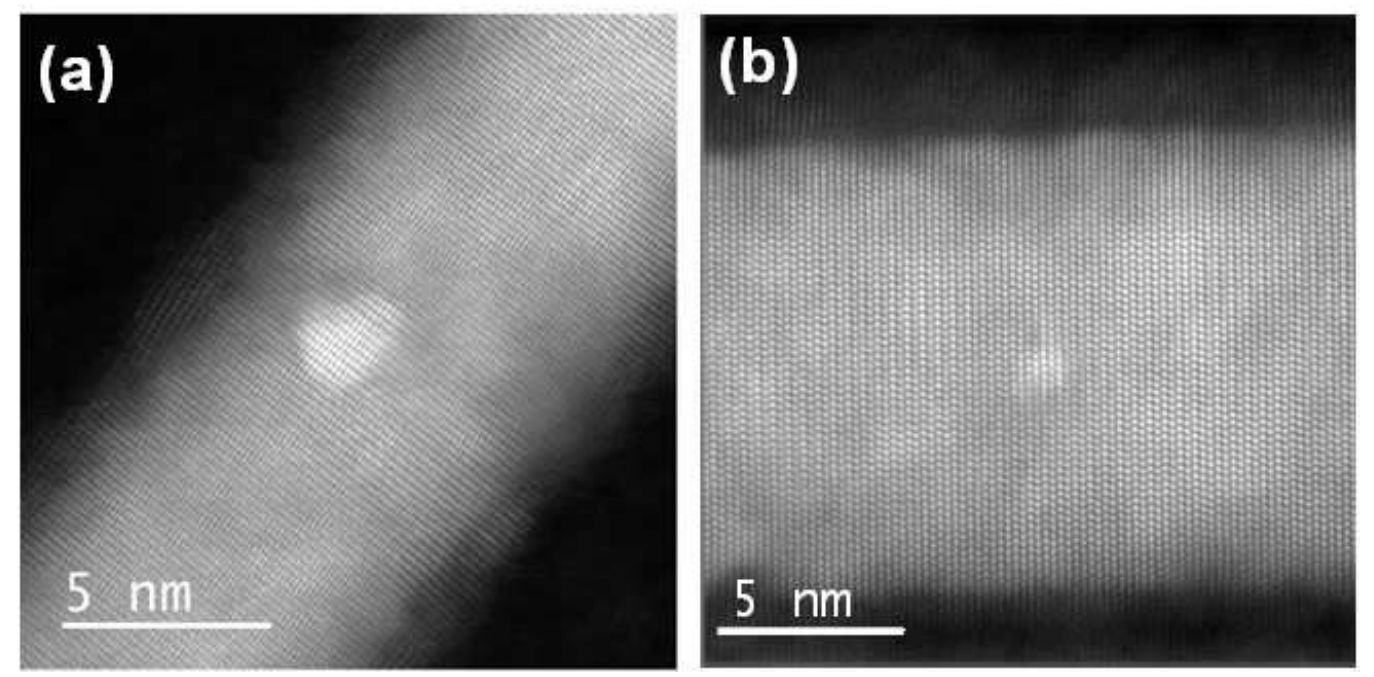

Figure S12. HAADF-STEM images of Ru seeds of $\mathrm{CO}_{\mathrm{Ru}}$ nanorods: (a) seed with irregular shape of ca. $1.9 \times 2.3 \mathrm{~nm}$ in size (nanorods prepared at $215^{\circ} \mathrm{C}$, heating rate of $25^{\circ} \mathrm{C} . \mathrm{min}^{-1}$ ); (b) isotropic seed with a diameter of c.a. $1.3 \mathrm{~nm}$ (nanorods prepared at $175^{\circ} \mathrm{C}$, heating rate of $8^{\circ} \mathrm{C} \cdot \mathrm{min}^{-1}$ ).

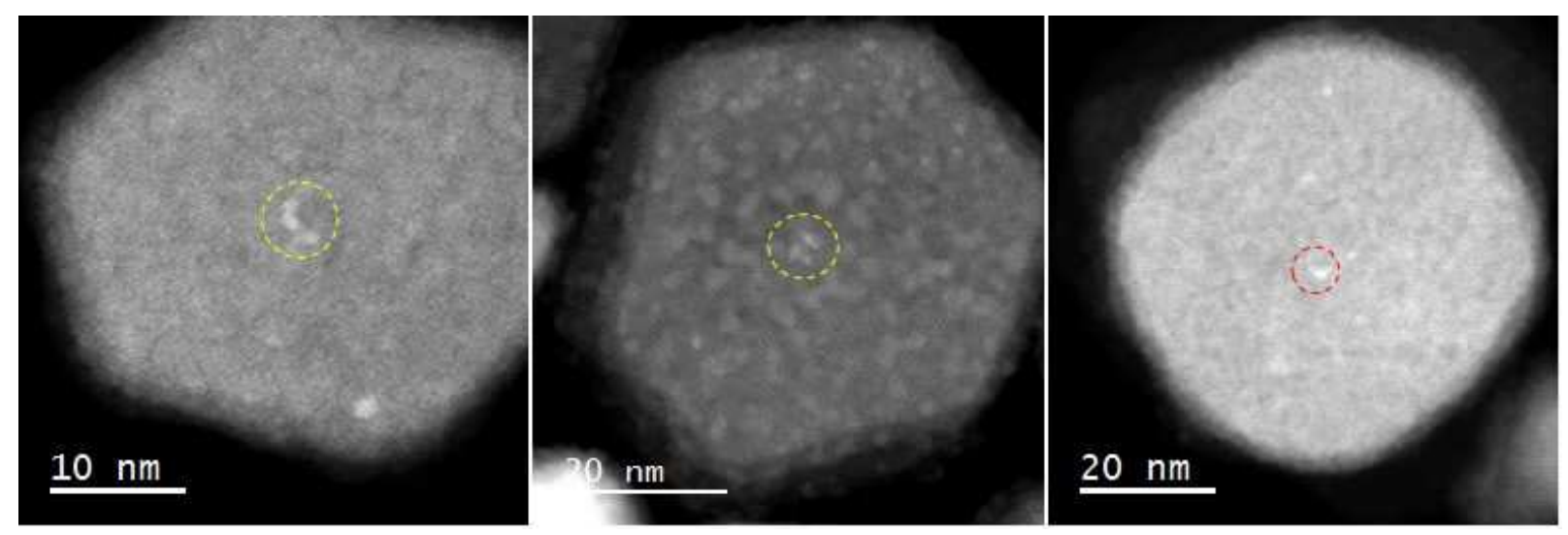

Figure S13. Different morphologies for the Ru seed of $\mathrm{CO}_{\mathrm{Ru}}$ platelets. 

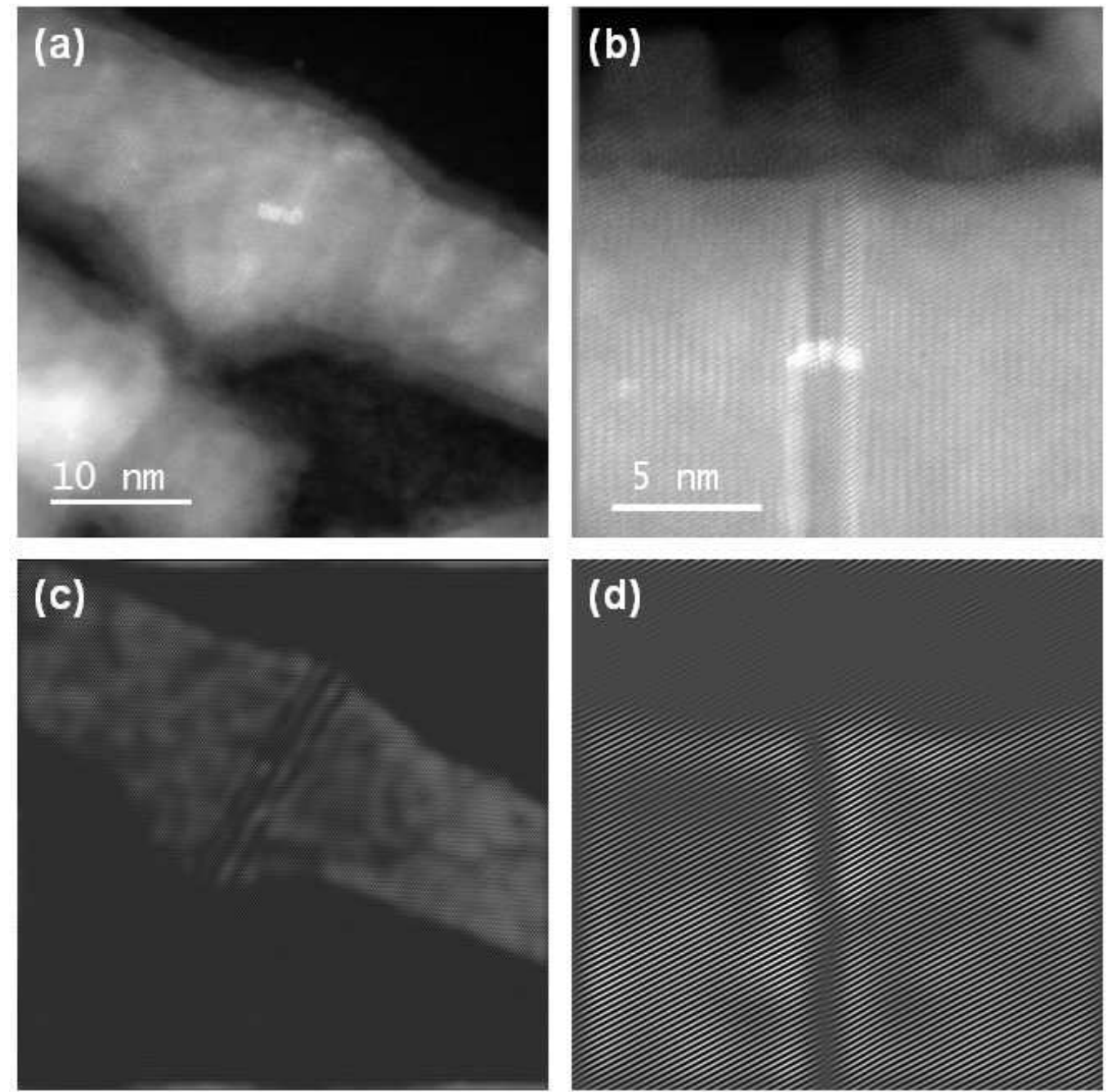

Figure S14. (a) and (b) HAADF-STEM images of Colr nanorods exhibiting structural defects around the Ir seeds ; (c) and (d) corresponding Fourier filtered images. 

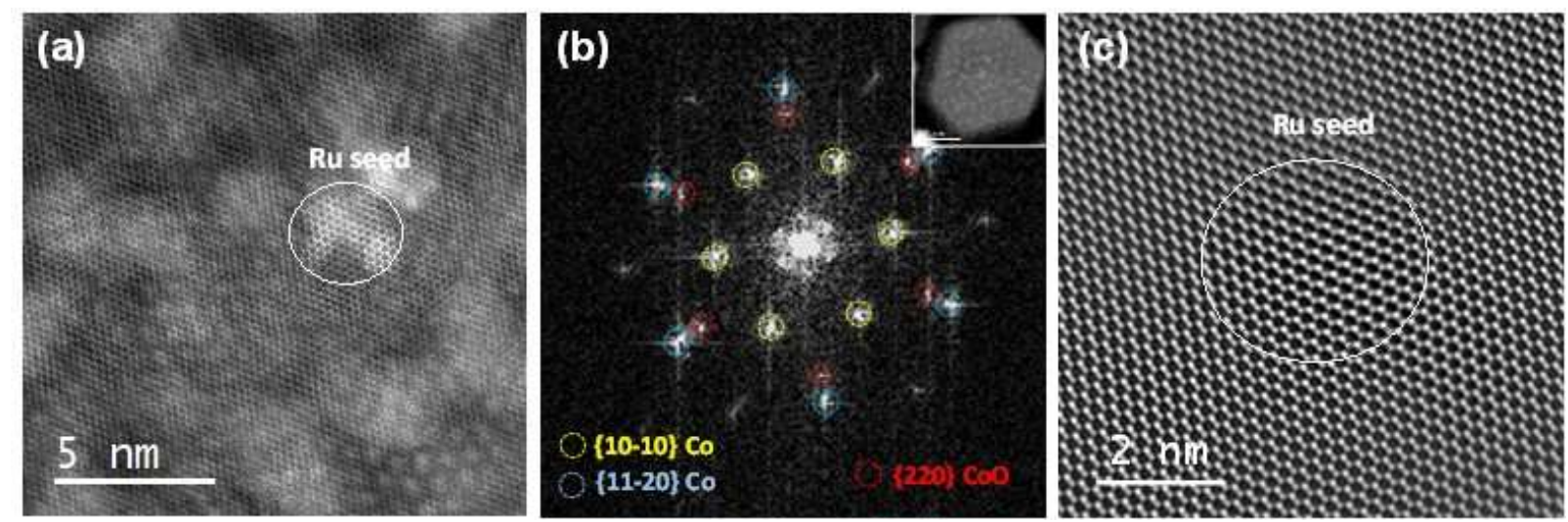

Figure S15. High resolution HAADF-STEM image of a Ru seed in a Co nanoplatelet ; (b) Fast Fourier transform indexed as hcp Co in [0001] zone axis (additional $\{220\}$ spots related to the CoO shell are also detected). Inset: Image of the platelet ; (c) Zoom on the Fourier filtered image selecting the $\{10 \overline{1} 0\}$ and $\{11 \overline{2} 0\}$ spots of the Co hcp structure. On top of the seed the $a$ parameter is 4 to $5 \%$ larger than outside the seed in agreement with the presence of hcp ruthenium with the same crystallographic orientation, in bulk metals the misfit between the interatomic spacing in the (0002) planes being $7.5 \%$. 
(a)

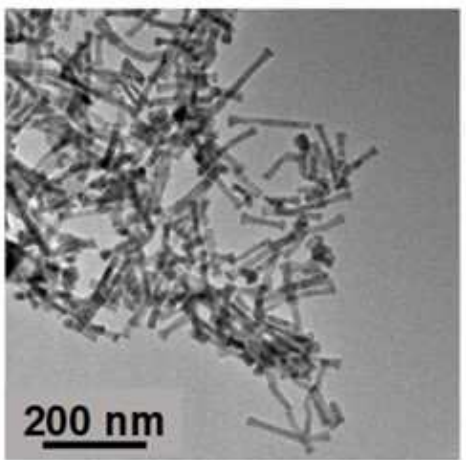

(b)

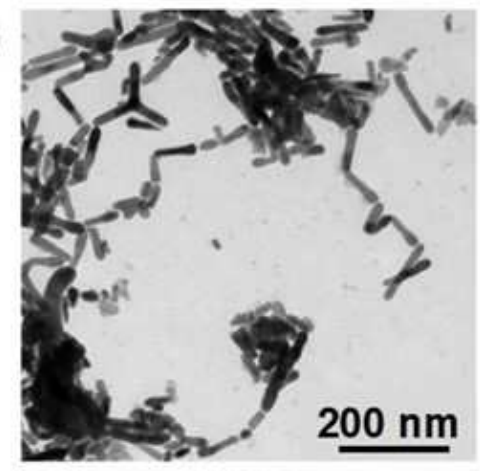

(c)

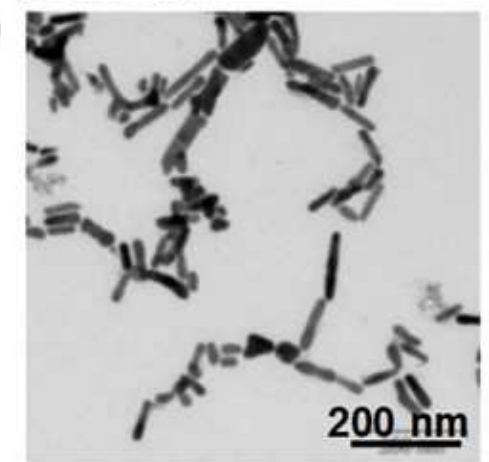

(d)

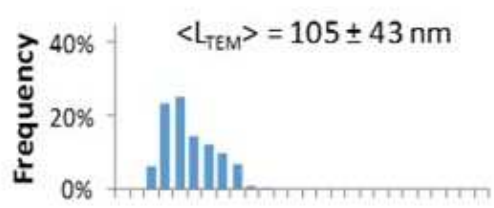

(e)

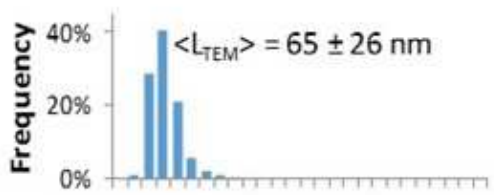

(f)

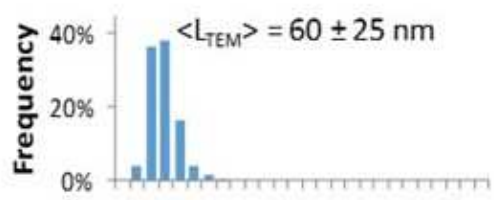

(g)

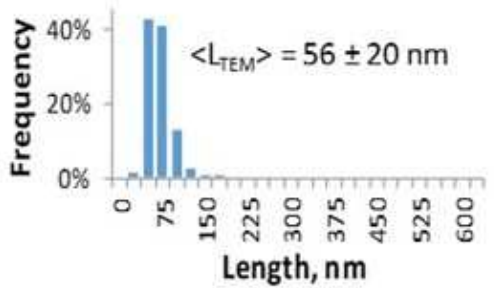

(h)

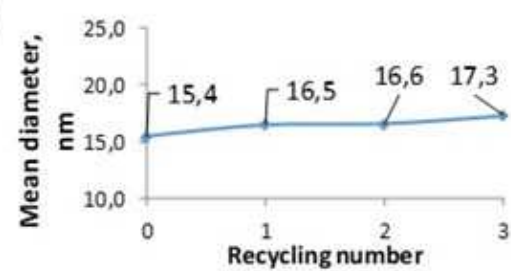

Figure S16. Variation of Coir nanorods morphology after repeated catalytic tests of conversion of octan2-ol into octan-2-one with molecular hydrogen production through an acceptorless dehydrogenation reaction. A suspension of CoIr NRs in octan-2-ol was heated to $453 \mathrm{~K}$ during $24 \mathrm{~h}$. The alcohol/Co molar ratio was fixed initially to $150 / 1$. For evaluation of the recyclability of the catalysts, the particles were recovered after $24 \mathrm{~h}$ of reaction using a permanent magnet, washed with the substrate and reused under the same operating conditions [from Ref. 1].

(a) TEM image of $\mathrm{Co}_{\text {Ir }}$ nanorods prepared by the polyol process; TEM images of the same rods after (b) a single catalytic test and (c) the catalytic test repeated three times; mean length of the Colr NRs : (d) after the synthesis; (e) after one test of $24 \mathrm{~h}$; (f) after two tests; (g) after three tests; (h) variation of the mean diameter before and after the catalytic tests.

It is noticeable that the mean length decreased by a factor of 2 after the first catalytic test and was then almost constant after subsequent tests. The mean diameter, on the other hand, remains constant during the repeated tests (see more details in Ref. 1).

${ }^{1}$ Viola, A.; Peboscq, M.; Peron, J.; Giraud, M.; Sicard, L.; Ramamoorthy, R. K.; Azeredo, B.; Nowak, S.; Decorse, P.; Viau, G.; Piquemal, J-Y. Catal. Today 2019, 333, 97-104. 

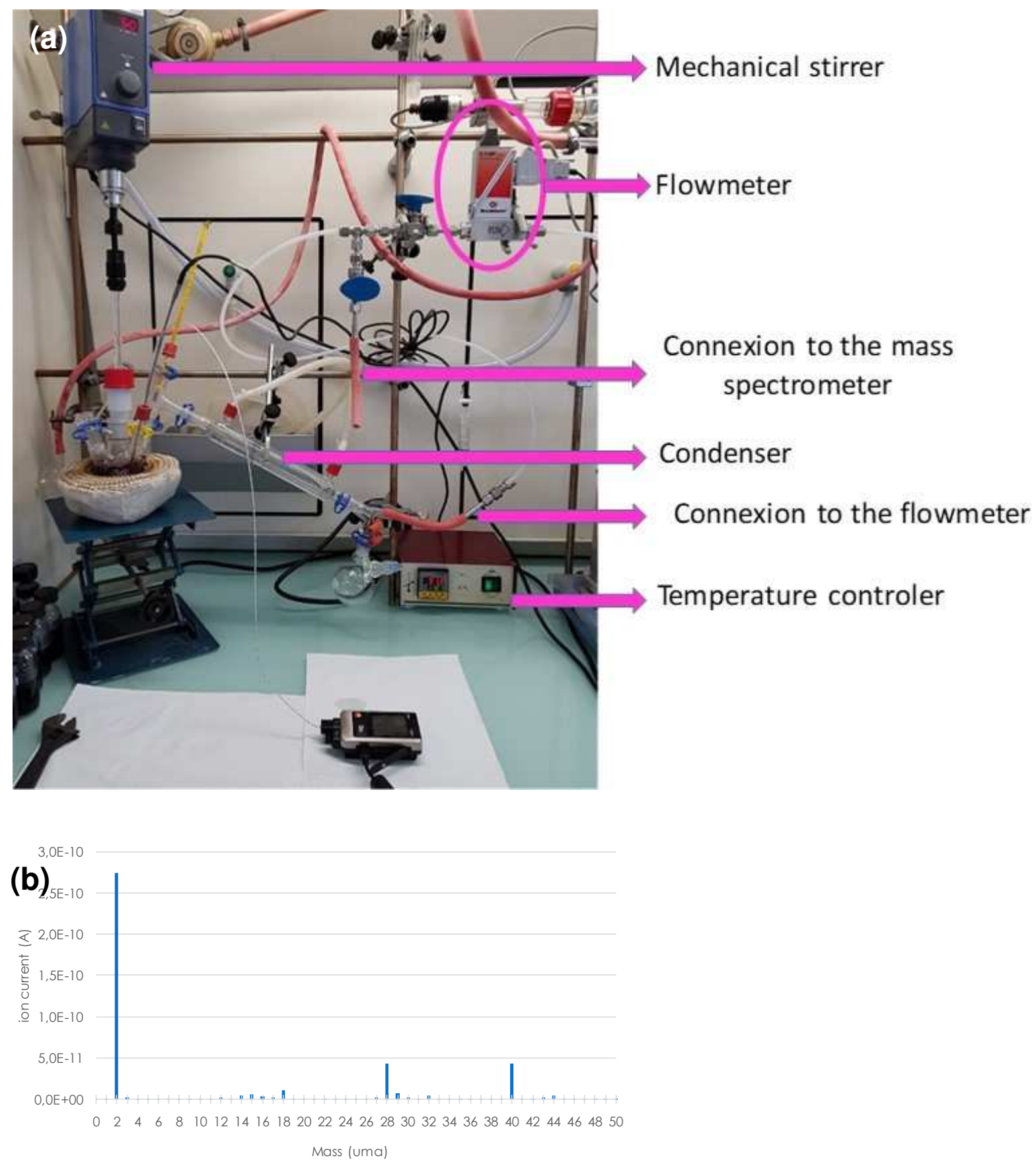

Figure S17. (a) Experimental set-up for the production of one-step SMG of cobalt nanoparticles with a flowmeter connected at the outlet of the condenser for in situ measurement of $\mathrm{H}_{2}$ flow; (b) Mass spectrum of a gas sample collected during a peak evolution showing a main peak at a mass of 2 uma corresponding to $\mathrm{H}_{2}$ (the others peak at 28 and 40 correspond to $\mathrm{N}_{2}$ and $\mathrm{Ar}$ used to flush the reactor). 

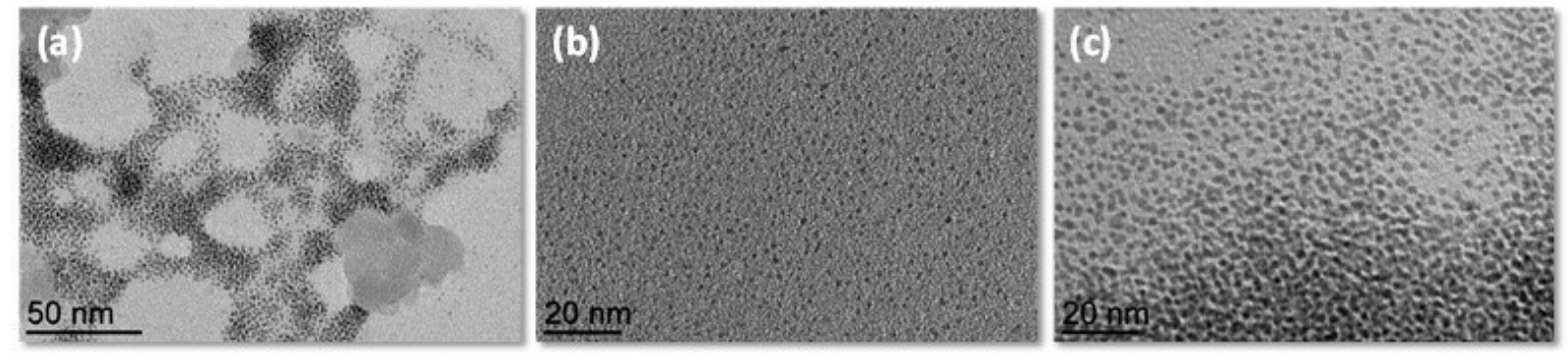

Figure S18. TEM images of (a) Ir nanoparticles synthesized by reduction of $\operatorname{IrCl}_{3} \cdot \mathrm{x}_{2} \mathrm{O}(2 \mathrm{mM})$ in a solution of $\mathrm{NaOH}(0.075 \mathrm{M}$ ) in 1,2-butanediol (mean size $=1.5 \mathrm{~nm}$ ); (b) Ru nanoparticles synthesized by reduction of $\mathrm{RuCl}_{3}(2 \mathrm{mM})$ in a solution of $\mathrm{NaOH}(0.075 \mathrm{M})$ in 1,2-butanediol (mean size $=1.3 \mathrm{~nm}$ ); (c) Ru nanoparticles synthesized by reduction of $\mathrm{RuCl}_{3}(2 \mathrm{mM})$ in a solution of sodium palmitate $(0.16 \mathrm{M})$ in 1,2-butanediol (mean size $=1.9 \mathrm{~nm}$ ). 


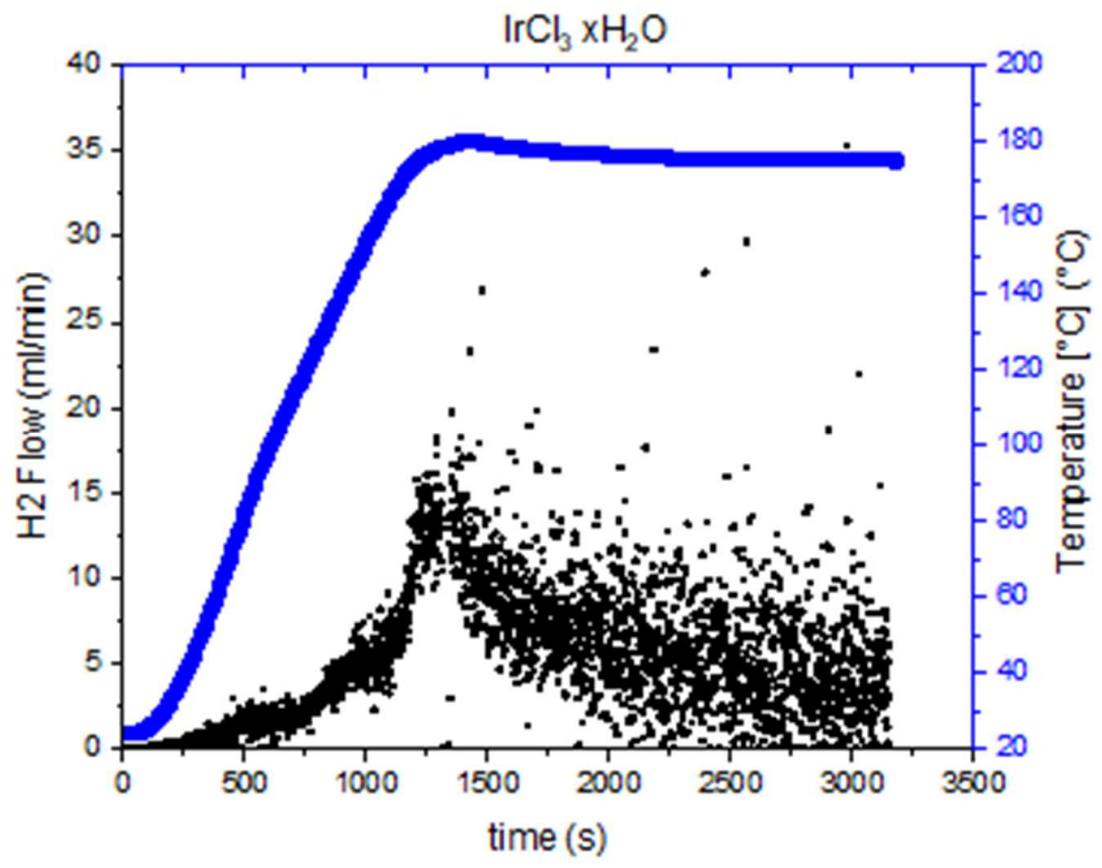

Figure S19. Reaction temperature and $\mathrm{H}_{2}$ flow as function of time measured during the reduction of 2 $\mathrm{mM}$ hydrated $\mathrm{IrCl}_{3}, \mathrm{xH}_{2} \mathrm{O}$ in a solution of $\mathrm{NaOH}$ in 1,2-butanediol. 


\section{REFERENCES}

(1) Wu, L.; Mendoza-Garcia, A.; Li, Q.; Sun, S. Chem. Rev. 2016, 116, 10473-10512.

(2) Jain, Prashant K.; Huang, X.; El-Sayed, I. H.; El-Sayed, M.A., Acc. Chem. Res. 2008, 41, 15781586.

(3) Kamyshny, A.; Magdassi, S. Small 2014, 10, 3515-3535.

(4) An, K.; Somorjai, G. A. ChemCatChem 2012, 4, 1512-1524.

(5) Wu, Z.; Yang, S.; Wu, W. Nanoscale 2016, 8, 1237-1259.

(6) Xia, Y.; Gilroy, K. D.; Peng, H.-C.; Xia, X. Angew. Chem. Int. Ed. 2017, 56, 60-95.

(7) Gawande, M. B.; Goswami, A.; Asefa, T.; Guo, H.; Biradar, A. V.; Peng, D.-L.; Zboril, R.; Varma, R. S. Chem. Soc. Rev. 2015, 44, 7540-7590.

(8) Zheng, Y.; Zhong, X.; Li, Z.; Xia, Y. Part. Part. Syst. Charact. 2014, 31, 266-273.

(9) Fiévet, F.; Lagier, J. P.; Blin, B.; Beaudoin, B.; Figlarz, M. Solid State lonics 1989, 32-33, 198-205.

(10) Toneguzzo, P.; Viau, G.; Acher, O.; Fiévet-Vincent, F.; Fiévet, F. Adv. Mater. 1998, 10, 10321035.

(11) Joseyphus, R. J.; Shinoda, K.; Kodama, D.; Jeyadevan, B. Materials Chemistry and Physics 2010, 123, 487-493.

(12) Viau, G.; Toneguzzo, P.; Pierrard, A.; Acher, O.; Fiévet-Vincent, F.; Fiévet, F. Scripta Materiala 2001, 44, 2263-2267.

(13) Jana, N. R., Gearheart, L., Murphy, C. J. Adv. Mater. 2001, 13, 1389-1393.

(14) Nikoobakht, B.; El-Sayed, M. A. Chem. Mater. 2003, 15, 1957-1962.

(15) Zhao, M.; Xu, L.; Vara, M.; Elnabawy, A. O.; Gilroy, K. D.; Hood, Z. D.; Zhou, S.; FigueroaCosme, L.; Chi, M.; Mavrikakis, M.; Xia, Y. ACS Catal. 2018, 8, 6948-6960.

(16) Niu, W.; Zhang, L.; Xu, G. Nanoscale 2013, 5, 3172-3181.

(17) Habas, S.E.; Lee, H.; Radmilovic, V.; Somorjai, G.A.; Yang, P. Nature Mater. 2007, 6, 692-697.

(18) Tebbe, M.; Kuttner, C.; Mayer, M. ; Maennel, M. ; Pazos-Perez, N ; A.F. König, T.; Fery, A. J. Phys. Chem. C 2015, 119, 9513-9523.

(19) Sánchez-Iglesias, A.; Winckelmans, N.; Altantzis, T.; Bals, S.; Grzelczak, M.; Liz-Marzán L. M. J. Am. Chem. Soc. 2017, 139, 107-110.

(20) Meng, M.; Fang, Z.; Zhang, C.; Su, H.; He, R.; Zhang, R.; Li, H.; Li, Z.-Y.; Wu, X.; Ma, C.; Zeng, J. Nano Lett. 2016, 16, 3036-3041.

(21) Luo, M.; Huang, H.; Choi, S. -I.; Zhang, C.; Rosa da Silva, R.; Peng, H.-C.; Li, Z.-Y.; Liu, J.; He, Z.; Xia Y. ACS Nano 2015, 9, 10523-10532. 
(22) Luo, M.; Ruditskiy, A.; Peng, H.-C.; Tao, J.; Figueroa-Cosme, L.; He, Z.; Xia Y. Adv. Funct. Mater. 2016, 26, 1209-1216.

(23) Laramy, C. R.; Fong, L. -K.; Jones, M. R.; O’Brien, M. N.; Schatz, G. C.; Mirkin C. A. Chem. Phys. Lett. 2017, 683, 389-392.

(24) Langille, M. R. ; Zhang, J. ; Personick, M. L. ; Li, S.; Mirkin, C. A. Science 2012, 337, 954-957.

(25) Abécassis, B. ; Testard, F. ; Spalla, O . ; Barboux, P. Nano Lett. 2007, 7, 1723-1727.

(26) Cormary, B.; Li, T.; Liakakos, N.; Peres, L.; Fazzini, P.-F.; Blon, T.; Respaud, M.; Kropf, A. J.; Chaudret, B.; Miller, J. T.; Mader, E. A.; Soulantica K. J. Am. Chem. Soc. 2016, 138, 8422-8431.

(27) Zhang, Y.; Liu, J.; Ahn, J.; Xiao, T.-H.; Li, Z.-Y.; Qin D. ACS Nano 2017, 11, 5080-5086.

(28) Carey, III, J. L.; Whitcomb, D. R.; Chen, S.; Penn, R. L.; Bühlmann P. ACS Nano 2015, 9, 12104-12114

(29) Watzky, M. A. and Finke R. G. J. Am. Chem. Soc. 1997, 119, 10382-10400.

(30) Xia, Y.; Xia, X.; Peng, H.-C. J. Am. Chem. Soc. 2015, 137, 7947-7966.

(31) Fiévet, F.; Ammar-Merah, S.; Brayner, R.; Chau, F.; Giraud, M.; Mammeri, F.; Peron, J.; Piquemal, J. Y.; Sicard, L.; Viau, G. Chem. Soc. Rev. 2018, 47, 5187-5233.

(32) Dong, H.; Chen, Y. C.; Feldmann, C. Green Chem. 2015, 17, 4107-4132.

(33) Liu, Q.; Guo, X.; Chen, J.; Li, J.; Song, W.; Shen, W. Nanotechnology 2008, 19, 365608.

(34) Gandha, K.; Elkins, K.; Poudyal, N.; Liu, X.; Liu, J. P. Sci. Reports 2014, 4, 5345.

(35) Pousthomis, M.; Anagnostopoulou, E.; Panagiotopoulos, I.; Boubekri, R.; Fang, W.; Ott, F.; Atmane, K. A.; Piquemal, J.-Y.; Lacroix, L.-M.; Viau, G. Nano Research 2015, 8, 2231-2241.

(36) Soumare, Y.; Piquemal, J. Y.; Maurer, T.; Ott, F.; Chaboussant, G.; Falqui, A.; Viau, G. J. Mater. Chem. 2008, 18, 5696-5702.

(37) Liu, Q.; Guo. X ; Wang, T.; Li, Y.; Shen, W. Mater. Lett. 2010, 64, 1271-1274.

(38) Gandha, K.; Poudyal, N.; Zhang, Q.; Ping Liu, J. IEEE Trans. Magn. 2013, 49, 3273-3276.

(39) Ouar, N.; Schoenstein, F.; Mercone, S.; Farhat, S.; Villeroy, B.; Leridon, B. ; Jouini, N. J. Appl. Phys. 2013, 114, 163907.

(40) Liébana-Viñas, S.; Wiedwald, U.; Elsukova, A.; Perl, J.; Zingsem, B.; Semisalova, A. S.; Salgueiriño, V. ; Spasova, M. ; Farle, M. Chem. Mater. 2015, 27, 4015-4022.

(41) Anagnostopoulou, E.; Grindi, B.; Lacroix, L. M.; Ott, F.; Panagiotopoulos, I.; Viau, G. Nanoscale 2016, 8, 4020-4029.

(42) Ener, S. ; Anagnostopoulou, E. ; Dirba, I. ; Lacroix, L. -M. ; Ott, F. ; Blon, T. ; Piquemal, J. Y. ; Skokov, K. P. ; Gutfleisch, O. ; Viau, G. Acta Materialia 2018, 145, 290-297.

(43) Liu, Q., Cao, X., Wang, T., Wang, C., Zhang, Q., Ma, L. RSC Advances 2015, 5, 4861-4871. 
(44) Viola, A.; Peboscq, M.; Peron, J.; Giraud, M.; Sicard, L.; Ramamoorthy, R. K.; Azeredo, B.; Nowak, S.; Decorse, P.; Viau, G.; Piquemal, J-Y. Catal. Today 2019, 333, 97-104.

(45) Harmel, J.; Peres, L.; Estrader, M.; Berliet, A.; Maury, S.; Fecant, A.; Chaudret, B.; Serp, P.; Soulantica, K. Angew. Chem., Int. Ed. 2018, 57, 10579-10583.

(46) González-Carballo, J. M.; Sadasivan, S.; Landon, P; Tooze, R. P. Mater. Charac., 2016, 118, 519-526.

(47) Mrad, K. ; Schoenstein, F. ; Nong, H. T. T. ; Anagnostopoulou, E. ; Viola, A. ; Mouton, L. ; Mercone, S. ; Ricolleau, C. ; Jouini, N. ; Abderraba, M. ; Lacroix, L.-M. ; Viau, G. ; J.-Y. Piquemal, CrystEngComm 2017, 19, 3476 - 3484.

(48) Viola, A.; Peron, J.; Kazmierczak, K.; Giraud, M.; Michel, C.; Sicard, L.; Perret, N.; Beaunier, P.; Sicard, M.; Besson, M.; Piquemal, J.-Y. Catalysis Science \& Technology 2018, 8, 562-572.

(49) Li, C.; Wu, Q.; Yue, M.; Xu, H.; Palaka, S.; Elkins, K.; Ping Liu, J. AIP Advances 2017, 7, 056229.

(50) Soumare, Y. ; Garcia, C. ; Maurer, T. ; Chaboussant, G. ; Ott, F. ; Fiévet, F. ; Piquemal, J.-Y.; Viau, G. Adv. Funct. Mater., 19 (2009) 1971-1977.

(51) Aït Atmane, K.; Michel, C.; Piquemal, J. Y.; Sautet, P.; Beaunier, P.; Giraud, M.; Sicard, M.; Nowak, S.; Losno, R.; Viau, G. Nanoscale 2014, 6, 2682-2692.

(52) Watt, J.; Yu, C.; Chang, S. L.; Cheong, S.; Tilley, R. D. J. Am. Chem. Soc. 2013, 135, 606-609.

(53) Treacy, M.M. Microscopy and Microanalysis 2011, 17, 847-858.

(54) jcpds file $n^{\circ} 00-005-0727$

(55) jcpds file $n^{\circ}$ 00-006-0598

(56) Bedford, N.; Dablemont, C.; Viau, G.; Chupas, P.; Petkov, V. J. Phys. Chem. C 2007, 111, 18214-18219.

(57) Hÿtch, M. J.; Snoeck, E.; Kilaas, R. Ultramicroscopy 1998, 74, 131-146.

(58) Crabtree, R. H. Chem. Rev. 2017, 117, 9228-9246.

(59) Nielsen, M.; Kammer, A.; Cozzula, D.; Junge, H.; Gladiali, S.; Beller M. Angew. Chem. Int. Ed. 2011, 50, 9593-9597.

(60) Kim, W.-H.; Park, I. S.; Park J. Org. Lett. 2006, 8, 2543-2545.

(61) Zhang, R. F.; Kong, X. F.; Wang, H. T.; Zhang, S. H.; Legut, D.; Sheng, S. H.; Srinivasan, S.; Rajan, K.; Germann, T. C. Sci. Rep. 2017, 7, 9577. 